Sukhyun Song is a Research Associate of the Computer Science Department at the University of Maryland, College Park. He received his PhD and MS degrees in Computer Science from the University of Maryland and his BS degree in Computer Science and Engineering from Seoul National University, Korea. Dr. Song's research interests include the performance optimization for parallel computing applications and the design and analysis of distributed infrastructure. 
Jeffrey K. Hollingsworth is a Professor of the Computer Science Department at the University of Maryland, College Park. He also has an appointment in the University of Maryland Institute for Advanced Computer Studies and the Electrical and Computer Engineering Department. He received his $\mathrm{PhD}$ and MS degrees in computer sciences from the University of Wisconsin. He received a B. S. in Electrical Engineering from the University of California at Berkeley. Dr. Hollingsworth's research seeks to develop a unified framework to understand the performance of large systems and focuses in performance measurement and auto tuning. He is Editor in chief of the journal Parallel Computing, was general chair of the SC12 conference, and is Vice Chair of ACM SIGHPC. 
*Biographies (Photograph)

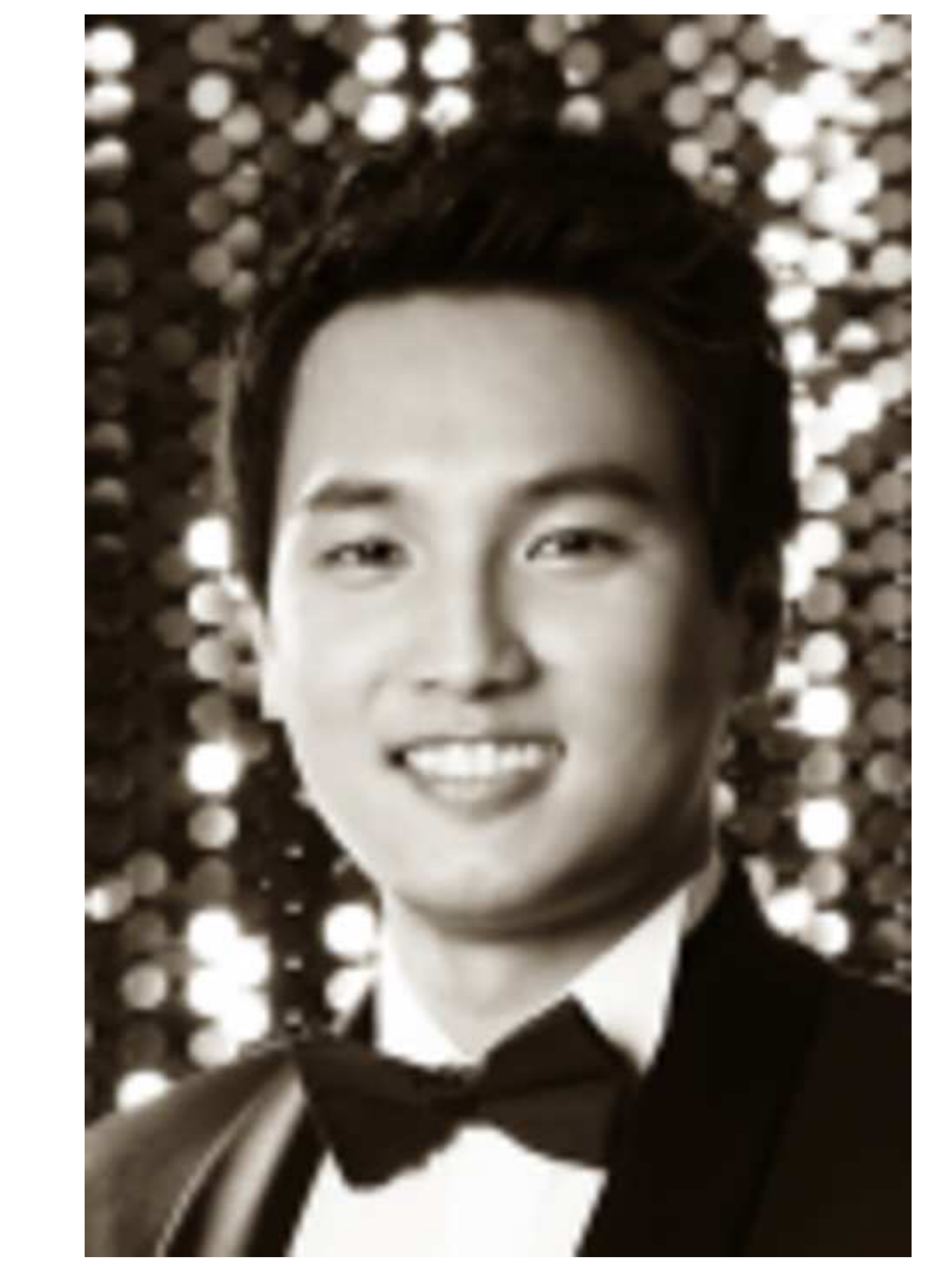

.

.

(1)

Blographies (Photograph)

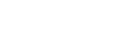

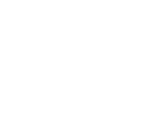

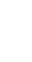

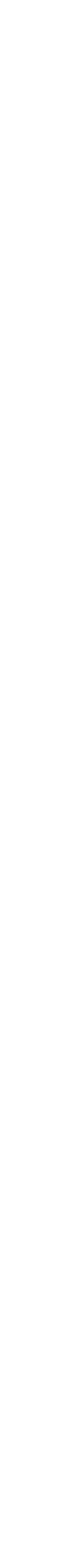




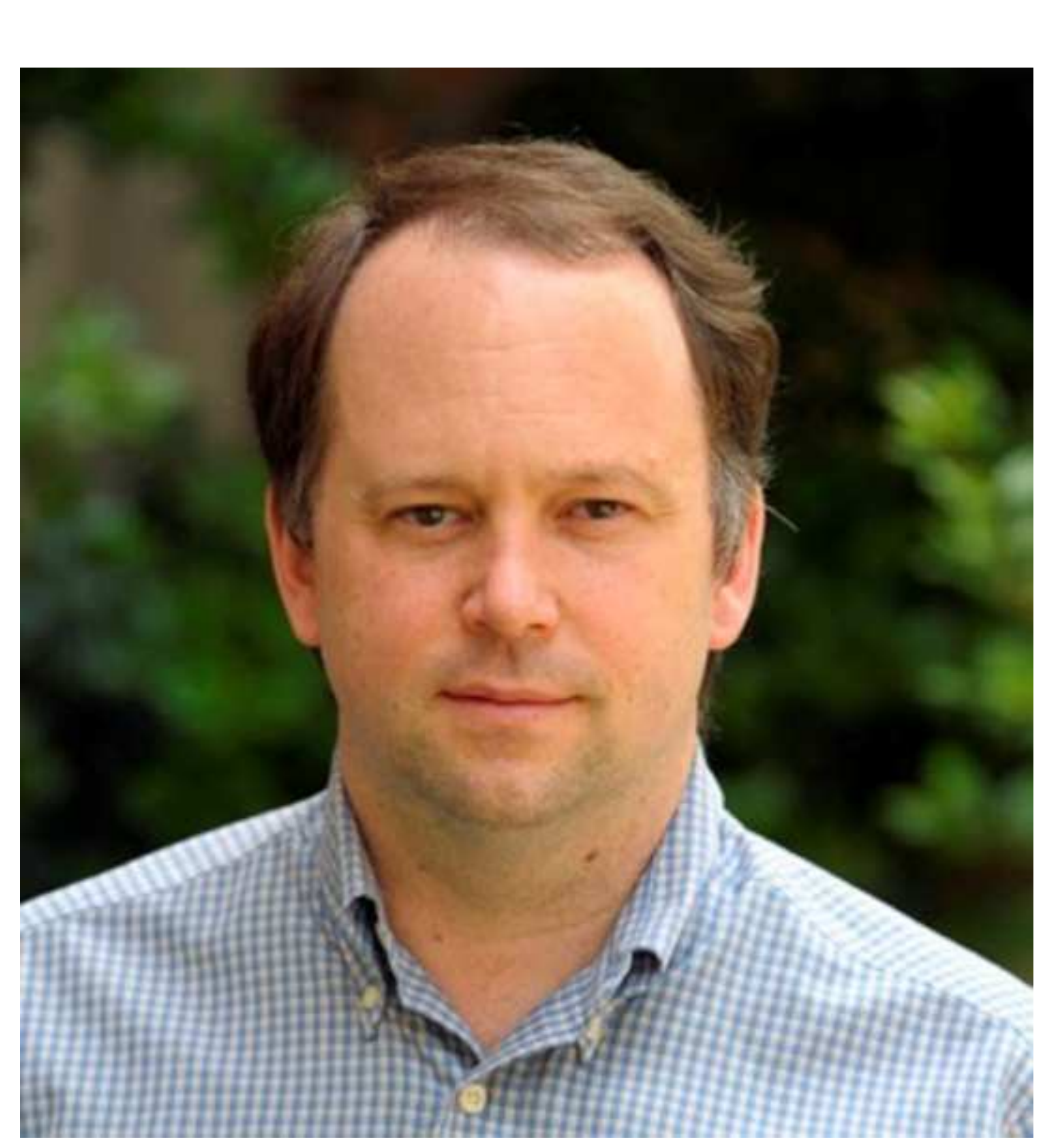

\section{${ }^{\star}$ Biographies (Photograph) h)}

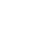

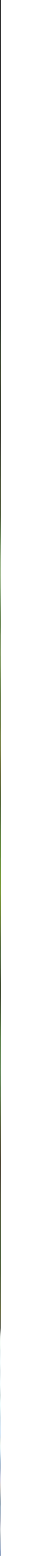

o

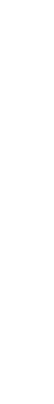

. 


\title{
Computation-Communication Overlap and Parameter Auto-Tuning for Scalable Parallel 3-D FFT
}

\author{
Sukhyun Song and Jeffrey K. Hollingsworth \\ Department of Computer Science, University of Maryland, College Park \\ $\{$ shsong, hollings\}@cs.umd.edu
}

\begin{abstract}
Parallel 3-D FFT is widely used in scientific applications, therefore it is important to achieve high performance on largescale systems with many thousands of computing cores. This paper describes a new method for scalable high-performance parallel 3-D FFT. We use a 2-D decomposition of 3-D arrays to increase scaling to a large number of cores. In order to achieve high performance, we use non-blocking MPI all-to-all operations and exploit computation-communication overlap. We also auto-tune our 3-D FFT code efficiently in a large parameter space and cope with the complex trade-off in optimizing our code in various system environments. According to experimental results from two systems, our method computes parallel 3-D FFT significantly faster than three existing libraries, and scales well to at least 32,768 compute cores.

Keywords: 3-D FFT; MPI; Non-Blocking Collective; Computation-Communication Overlap; Auto-Tuning
\end{abstract}

\section{Introduction}

It is important to achieve high performance of the Fast Fourier Transform (FFT) as FFT is widely used in many fields of science and engineering. For example, researchers have recently used three-dimensional FFT (3-D FFT) to run astrophysical $N$-body simulations [1] and turbulent flow simulations [2] on high-performance computing systems. Computing 3-D FFT requires a large number of floating point and memory access operations. So we need a parallel algorithm that can be run on a large-scale system. However, parallel 3-D FFT requires expensive allto-all communication. Each parallel computing process exchanges a 3-D array with all the other processes. We believe that it would be beneficial to overlap expensive computation and communication in achieving high performance of parallel 3-D FFT.

In this paper, we present a new method for parallel 3-D FFT that can increase scaling to a large number of computing cores in a distributed-memory parallel system, and exploits computation-communication overlap. $1 \mathrm{We}$ design a scalable method by following the 2-D domain decomposition technique [4, 5, 6, 7, 8] that decomposes a 3-D array along two dimensions. Accordingly, we can use more computing cores than the approaches presented in 9, 10, 11] that use the 1-D domain decomposition and only decompose an array along one dimension. We exploit the overlap of computation and communication by

${ }^{1}$ Note that this paper is an extended version of our previous paper [3], so the two papers share several figures and texts. using the non-blocking MPI_Ialltoall operation that is described in the MPI-3.0 standard 12]. As the MPI library is widely used in the parallel computing community, it is important to design a parallel 3-D FFT code based on MPI and achieve portability. We use collective communication instead of point-to-point communication due to its better performance and simplicity. The MPI collectives are optimized for complex communication patterns among parallel processes, and we can write a simple code based on collective operations.

Our work is the first to effectively apply non-blocking MPI collectives to the 2-D decomposition technique for parallel 3-D FFT. Popular FFT libraries [4, 5, 9] do not exploit non-blocking communication. Although the work by Nishtala et al. 8] overlaps computation and communication, it is written in UPC and based on point-to-point communication. Hoefler et al.'s work [10] and our previous work 11] use MPI_Ialltoall, but their scalability is limited as they use a 1-D domain decomposition.

Our strategy for computation-communication overlap is to have each process divide an array into multiple small blocks, and repeat computation and non-blocking communication on the divided blocks. In this way, we have opportunities to overlap computation on one block with communication on other blocks, and eventually increase the overall performance. We also increase scaling by applying our overlap strategy to each of the two communication phases in the 2-D decomposition technique for parallel 3-D FFT.

To optimize the overall performance of our method, we identify and resolve the following requirements. First, all possible computation steps should be used for the overlap. 
Parallel 3-D FFT involves three steps of pre-computation, communication, and post-computation. Simpler approaches given in $[8,10,13]$ can only overlap pre-computation on one data block with communication on other blocks, then perform post-computation on the entire blocks. Instead, we have both pre-computation and post-computation overlapped with communication. Second, computation should be balanced between the two communication phases. The 2-D decomposition method involves two phases of all-toall communication, and there exist three local computation steps before and after each phase. When the communication time is different between two phases, we divide the computation in the middle into two. We then assign a longer computation to the phase with the longer communication. In this way, we increase the opportunity to overlap the computation in the middle with communication. Third, code should be portable. One way to ensure fully asynchronous communication for non-blocking MPI collectives is to offload communication processing to special hardware. Instead, we choose another option for asynchronous communication, which calls MPI_Test periodically, due to its greater portability. Last, we should automatically handle the complex trade-off regarding our optimization techniques. We parameterize our parallel 3D FFT code and auto-tune the parameters. For example, a data block size for communication and a MPI_Test call frequency are set by tunable parameters. Since the parameter space is very large (more than trillions of possible configurations), hand-tuning is not feasible. We utilize the publicly available Active Harmony auto-tuning library [14] and find a good parameter configuration efficiently. We also introduce several techniques to tune our FFT code fast and effectively.

We conducted experiments on two systems at NERSC using up to 32,768 compute cores. Our code performs parallel 3-D FFT faster than three other approaches. Specifically, we achieve a speedup by up to $1.83 \times$ over the FFTW library [9], 2.57 $\times$ over the 2DECOMP\&FFT library [5], and $1.95 \times$ over the UPC-based code [8].

The rest of the paper is organized as follows. We first describe background information in Section 2 Sections 3 and 4 present how we design and auto-tune our parallel 3 -D FFT code. We relate our evaluation and results in Section 5 Section 6 provides the related work. Finally, we conclude and discuss future work in Section 7.

\section{Background}

This section first reviews FFT computation and describes several basic assumptions underlying our design. We then introduce a general method for parallel 3-D FFT without any computation-communication overlap.

\subsection{Fast Fourier Transform}

With an input array $X$ of $N$ complex numbers, a onedimensional FFT produces an output array $Y$ of $N$ complex numbers. When $\omega_{N}=e^{-\frac{2 \pi}{N} i}, Y[k]$ is defined for

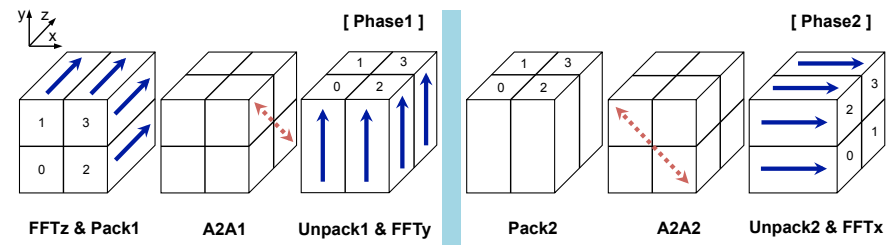

Figure 1: 2-D Domain Decomposition for Parallel 3-D FFT: An input 3 -D array is divided along $x$ and $y$ dimensions. There are two phases of blocking all-to-all communication on sub-groups of processes.

$k=0,1, \ldots, N-1$ as $Y[k]=\sum_{j=0}^{N-1} X[j] \omega_{N}^{j k}$. The $d-$ dimensional FFT can be computed simply as the composition of a sequence of $d$ sets of 1-D FFTs along each dimension. For example, 3-D FFT for $N^{3}$ complex numbers can be computed by three sets of $N^{2} 1$-D FFTs along each dimension.

We make the following assumptions in this paper. First, we focus on the forward transform that transforms $X$ into $Y$. Our approach can be easily applied to transform $Y$ backward into $X$. Second, we only describe the complexto-complex transform that takes an input array of complex numbers and produces an output array of complex numbers. We have also implemented the real-to-complex transform by applying our computation-communication overlap methods, but omit the details of this implementation due to space limitations. Last, we focus on the in-place transform and want the output to overwrite the input array. Our approach can be applied directly for the out-of-place transform where the output is written to a separate array.

\subsection{2-D Domain Decomposition for Parallel 3-D FFT}

Our design for a parallel 3-D FFT basically follows the 2-D domain decomposition method. The overall procedure of the 2-D domain decomposition method consists of several steps as shown in Figure 1] We parallelize 3-D FFT with $\boldsymbol{p}$ processes, and Figure 1 shows an example of $p=4$. An input 3 -D array is divided equally into $\boldsymbol{p}_{\mathbf{1}}$ subarrays along the $x$ dimension and $\boldsymbol{p}_{\mathbf{2}}$ sub-arrays along the $y$ dimension. $p_{1}=2$ and $p_{2}=2$ in Figure 1 and process numbers are marked on each sub-array. Then each process executes the following steps on each sub-array:

1. FFTz: Compute 1-D FFTs along the $z$ dimension. (Assume that elements on the $z$ dimension are adjacent in memory.)

2. Pack1: Pack the 3-D sub-array data into a buffer in preparation for all-to-all communication.

3. A2A1: Perform blocking all-to-all communication for each group of $p_{2}$ processes.

4. Unpack1: Unpack the received data into the 3-D sub-array with the new memory layout such that elements on the $y$ dimension are adjacent in memory.

5. FFTy: Compute 1-D FFTs along the $y$ dimension.

6. Pack2: Pack the 3-D sub-array data into a buffer in preparation for all-to-all communication. 


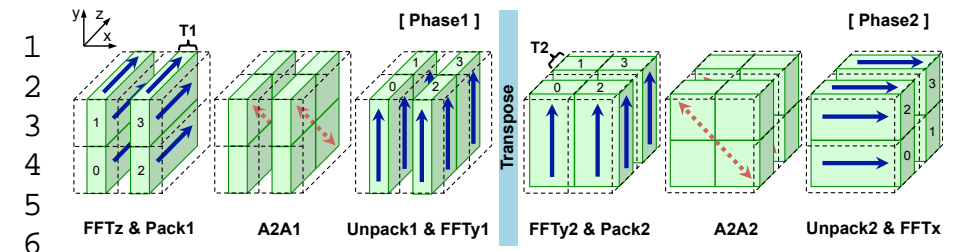

Figure 2: Parallel 3-D FFT with Computation-Communication Overlap: A sub-array is divided again into communication tiles. We 9 repeat computation and non-blocking communication on the com10 munication tiles.
$N_{y}$, and $N_{z}$ elements (complex numbers) on the $x, y$, and $z$ dimensions, respectively. Each of $p=p_{1} \times p_{2}$ processes is assigned a sub-array of $\frac{N_{x}}{p_{1}} \times \frac{N_{y}}{p_{2}} \times N_{z}$ elements. The 3-D FFT performance can depend on how $p$ is decomposed into $p_{1} \times p_{2}$. So we introduce a decomposition parameter $\boldsymbol{D}$ to represent the value of $p_{1}$. Section 4 will discuss the method to auto-tune $D$ and other parameters of our $3-\mathrm{D}$ FFT code. The memory layout for the sub-array starts with $x-y-z$ in the row-major order. The elements on the $z$ dimension are adjacent in memory.

We divide the FFTy step in Figure 1 into two steps of FFTy1 and FFTy2 as shown in Figure 2. The computation amount of each divided step is determined by a parameter $\boldsymbol{R}_{\boldsymbol{y}}$ that is an integer number ranging from 0 through 10. FFTy1 performs 1-D FFT computation on $\frac{R_{y}}{10}$ of the total $1-\mathrm{D}$ arrays along the $y$ dimension during Phase1, and we finish the rest of 1-D FFT at the FFTy2 step in Phase2. By adjusting $R_{y}$ properly, we can balance the FFTy computation between two communication phases and have a good chance to optimize the overlap. For example, suppose that communication in each phase takes time longer than one 1-D FFT step but shorter than two. If we perform the whole FFTy step in Phase1 like in Figure 1] a part of computation cannot be overlapped with communication. In this case, we divide the FFTy step and distribute it over two phases, so that all 1-D FFT computation can be overlapped with communication.

To fulfill the overlap during Phase1, we divide a 3-D sub-array into multiple small blocks along the $x$ dimension as shown in Figure 2 We call each divided block a communication tile. We define a tile size parameter $\boldsymbol{T}_{\mathbf{1}}$ to handle the trade-off between the overlap efficacy and the messaging efficiency. With small $T_{1}$, we can overlap many computations with communication but there would be a large overhead of exchanging many small-sized messages. If $T_{1}$ gets bigger, there will be less overlap but higher efficiency for communication. Thus we should find a good value of $T_{1}$ to achieve high performance. Each tile contains $T_{1}$ elements on the $x$ dimension. So the number of elements in each communication tile is equal to $T_{1} \times \frac{N_{y}}{p_{2}} \times N_{z}$. We also define a window size parameter $\boldsymbol{W}_{\mathbf{1}}$ to specify the degree of communication parallelism. It is also important to adjust $W_{1}$ properly to utilize as many concurrent communication connections as possible.

Algorithm 1] describes a pseudocode for each process. Each process repeats the FFTz, Pack1, A2A1, Unpack1, and FFTy1 steps for each communication tile. Unlike the original 2-D decomposition method, Algorithm 11 uses the non-blocking MPI all-to-all operation (MPI_Ialltoall and MPI_Wait) for the A2A1 step. Thus we can overlap computation (FFTz, Pack1, Unpack1, and FFTy1) on one communication tile with communication (A2A1) for other tiles. After the Unpack1 step, the data in each communication tile are rearranged in memory to the $x-z-y$ order, so that we can execute the FFTy1 step on 1-D arrays along the $y$ dimension. To achieve high performance for the 1-D FFT computation steps, we utilize the highly optimized 


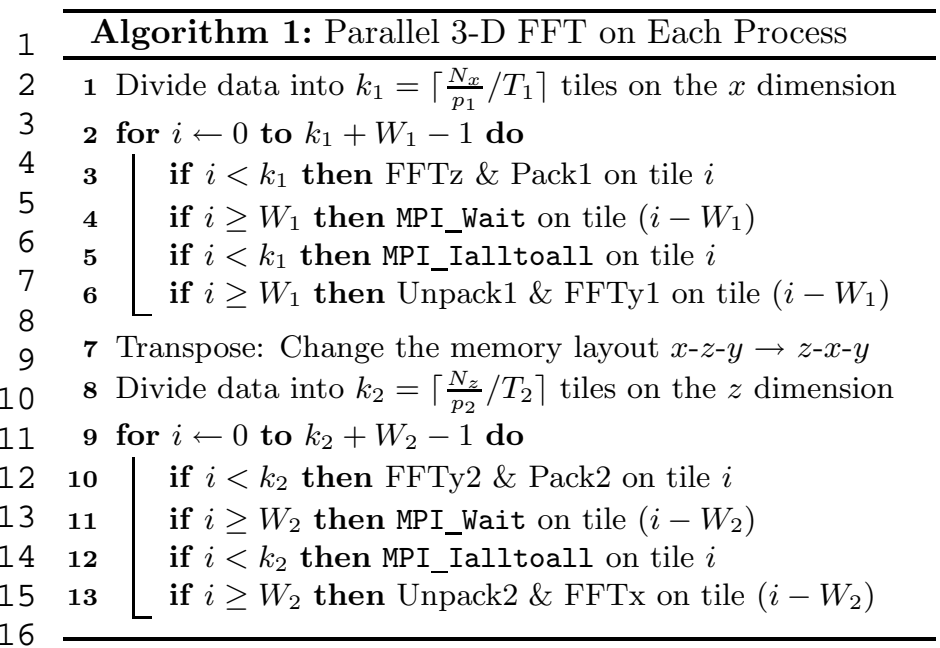

17

18

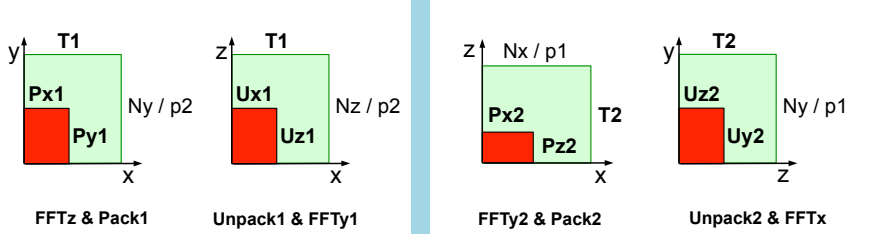

Figure 3: Loop Tiling for Cache Reuse: A communication tile is divided again into sub-tiles. We can increase cache hits during local computation by reading the sub-tile information from a cache.

It is important to determine the proper frequency of MPI_Test calls. Too high frequency will incur unnecessary function call overhead, and too low frequency will limit the progress of the all-to-all communication. To cope with the trade-off, we have four tunable parameters to adjust the frequency of MPI_Test calls during Phase1. $\boldsymbol{F}_{\boldsymbol{z}}$ defines the number of MPI_Test calls during FFTz for one communication tile. $\boldsymbol{F}_{\boldsymbol{p} \mathbf{1}}$ is the frequency parameter during the Pack1 step for one communication tile. $\boldsymbol{F}_{\boldsymbol{u} \boldsymbol{1}}$ is for Unpack1, and $\boldsymbol{F}_{\boldsymbol{y} \mathbf{1}}$ is for FFTy1. Likewise, we introduce $\boldsymbol{F}_{\boldsymbol{y} \mathbf{2}}$, $\boldsymbol{F}_{\boldsymbol{p} \mathbf{2}}, \boldsymbol{F}_{\boldsymbol{u} \boldsymbol{2}}$, and $\boldsymbol{F}_{\boldsymbol{x}}$ for FFTy2, Pack2, Unpack2, and FFTx in Phase2.

\subsection{Loop Tiling for Cache Reuse}

We optimize the FFTz and Pack1 steps by tiling the loop inside each communication tile. As shown in the left side of Figure 3 each communication tile is divided again into sub-tiles with $\boldsymbol{P}_{\boldsymbol{x} \boldsymbol{1}}$ elements on the $x$ dimension and $\boldsymbol{P}_{\boldsymbol{y} \mathbf{1}}$ on the $y$ dimension. So each sub-tile contains $P_{x 1} \times P_{y 1} \times N_{z}$ elements. By executing FFTz and Pack1 over each sub-tile, we can increase cache hit rate during Pack1. Similarly to FFTz and Pack1, we optimize Unpack1 and FFTy1 through loop tiling. Each communication tile is divided into sub-tiles with $\boldsymbol{U}_{\boldsymbol{x} 1}$ elements on the $x$ dimension and $\boldsymbol{U}_{\boldsymbol{z} \mathbf{1}}$ on $z$ dimension. Thus, we can increase cache hits during FFTy1 by reading the sub-tile information from a cache after Unpack1. Likewise, we utilize loop tiling in the second phase as shown in the right side of Figure 3] $\boldsymbol{P}_{\boldsymbol{x} \mathbf{2}}$ and $\boldsymbol{P}_{\boldsymbol{z} \mathbf{2}}$ are used for FFTy2 and Pack2, and $\boldsymbol{U}_{\boldsymbol{y} \mathbf{2}}$ and $\boldsymbol{U}_{\boldsymbol{z} \mathbf{2}}$ are used for Unpack2 and FFTx.

\subsection{Improvement Opportunities}

First, there is an opportunity to improve the performance by using MPI_Ialltoallv. If a 3 -D array is uniformly decomposed among all $p=p_{1} \times p_{2}$ parallel processes, four conditions must be satisfied: 1) $N_{x} \bmod p_{1}=$ $\left.0,2) N_{y} \bmod p_{1}=0,3\right) N_{y} \bmod p_{2}=0$, and 4) $N_{z} \bmod$ $p_{2}=0$. In the case of uniform domain decomposition, we can simply use MPI_Ialltoall as every process sends and receives the same amount of data. However, when any of the four conditions is not satisfied, the array cannot be uniformly decomposed. In the case of non-uniform domain decomposition, we have two options of all-to-all communication: 1) use MPI_Ialltoallv and allow each process to send a different amount of data or 2) use MPI_Ialltoall 
Table 1: 24 Auto-Tunable Parameters of Parallel 3-D FFT

$D \quad$ \# processes on $x$-dim

$T_{1} \quad$ \# elements on $x$-dim in a communication tile (Phase1)

$W_{1} \quad \max \#$ tiles for concurrent communication (Phase1)

$P_{x 1} \quad$ \# elements on $x$-dim in a sub-tile during Pack1

$P_{y 1} \quad$ \# elements on $y$-dim in a sub-tile during Pack1

$F_{z} \quad$ \# MPI_Test calls during FFTz for one tile

$F_{p 1} \quad \#$ MPI_Test calls during Pack1 for one tile

$U_{x 1} \quad$ \# elements on $x$-dim in a sub-tile during Unpack1

$U_{z 1} \quad$ \# elements on $z$-dim in a sub-tile during Unpack1

$F_{u 1} \quad$ \# MPI_Test calls during Unpack1 for one tile

$F_{y 1} \quad$ \# MPI_Test calls during FFTy1 for one tile

$R_{y} \quad$ computation ratio of FFTy1 to total FFTy

$T_{2} \quad$ \# elements on $z$-dim in a communication tile (Phase2)

$W_{2} \quad \max \#$ tiles for concurrent communication (Phase2)

$P_{z 2} \quad$ \# elements on $z$-dim in a sub-tile during Pack2

$P_{x 2} \quad$ \# elements on $x$-dim in a sub-tile during Pack2

$F_{y 2} \quad$ \# MPI_Test calls during FFTy2 for one tile

$F_{p 2}$ \# MPI_Test calls during Pack2 for one tile

$U_{z 2}$ \# elements on $z$-dim in a sub-tile during Unpack2

$U_{y 2} \quad$ \# elements on $y$-dim in a sub-tile during Unpack2

$F_{u 2}$ \# MPI_Test calls during Unpack2 for one tile

$F_{x} \quad$ \# MPI_Test calls during FFTx for one tile

$V \quad$ switch to use MPI_Ialltoallv or not

$S \quad$ switch to use the stride method or not

after padding messages. We introduce a parameter $\boldsymbol{V}$ to decide which option we should use for non-uniform domain decomposition. If $V=0$, we do not use MPI_Ialltoallv. If $V=1$, we use MPI_Ialltoallv only for A2A2. If $V=2$, we use MPI_Ialltoallv only for A2A1. If $V=3$, we use MPI_Ialltoallv for both A2A1 and A2A2.

The second opportunity is to use the stride method for 1-D FFT. FFTW allows us to perform 1-D FFT on an array with stride of general $N$ elements. If we utilize the stride method, we do not have to rearrange a memory layout for 1-D FFT computation. We can modify each step of our algorithm to maintain the $x-y-z$ layout. Then we have an opportunity to remove the Transpose step. We introduce a switch parameter $\boldsymbol{S}$ to decide whether to use the stride method $(S=1)$ or not.

As the last improvement technique, we remove the Transpose step when $N_{x}=N_{y}$. We modify Unpack2 to generate the $y-z-x$ layout (as opposed to $z-y-x$ ) for the case of $N_{x}=N_{y}$. Then the source and destination data for the A2A2 step will reside in the same memory area. Thus we can avoid the cost of the Transpose step and improve the overall 3-D FFT performance. We have no parameter for the third improvement technique because we always use it for the case of $N_{x}=N_{y}$.

\section{Auto-Tuning}

Table 1 summarizes the 24 tunable parameters contained in our parallel 3-D FFT code. Since it is impossible to investigate all the configurations manually and exhaustively, we need an efficient way to tune the parameters

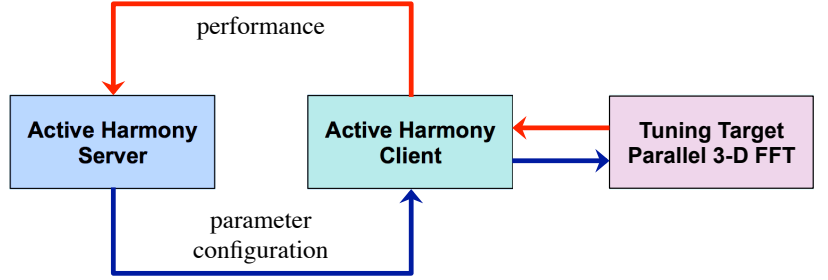

Figure 4: Auto-Tuning Procedure: The Active Harmony server decides a test parameter configuration. The Active Harmony client executes 3-D FFT with the received configuration, and reports the performance back to the server.

automatically and determine a good configuration. This section introduces an auto-tuning software framework and presents how we customize the auto-tuner for fast and effective tuning.

\subsection{Active Harmony}

We use a general software framework called Active Harmony (AH) [14] for auto-tuning because it provides easyto-use APIs to auto-tune user-specified parameters. Figure 4 shows the overall procedure of how $\mathrm{AH}$ interacts with our parallel 3-D FFT code. The AH server searches efficiently through a large parameter space and decides a parameter configuration to be tested on the tuning target (our parallel 3-D FFT code). The AH client receives a parameter configuration from the server, executes the tuning target with the received configuration, and reports the performance back to the server. This procedure is repeated until the $\mathrm{AH}$ server finds the best parameter configuration.

Among several strategies provided by the AH server, we use the Nelder-Mead (NM) method [16] to search for a good parameter configuration. NM is a commonly used optimization technique in many fields of science. NM uses the concept of a simplex, which is a polytope of $(d+1)$ vertices in $d$ dimensions. For example, a simplex is a triangle in two dimensions. The AH client measures the performance of 3-D FFT at each point (or parameter configuration) on a simplex. NM generates a new test point by extrapolating the performance values measured at points on the simplex. Then NM replaces one of the simplex points with the new point. For example, NM sometimes replaces the worst simplex point $W$ with a new point $R$ such that $R$ is reflected from $W$ through the centroid of the remaining $d$ points. The search procedure finishes when all the points on a simplex are close to each other, and can be considered to be converged to a single point. Further details about NM are described in [16].

\subsection{Initial Simplex}

An initial simplex in NM can affect the tuning time and the quality of the tuning result because a poor initial simplex could cause the search to get stuck at local minimum points. This section describes how we determine an initial simplex, and Section [5] will demonstrate its performance with experiments. We construct an initial simplex 
1 by choosing 25 random points within a limited area of pa-

2 rameter space. In other words, we choose a random value

3 for each parameter within a limited range. $D$ is chosen

4 from any number by which $p$ is divisible. $T_{1}$ and $T_{2}$ are

5 set, so that the number of elements of each tile ranges in

$6\left[2^{6}, 2^{22}\right]$. Therefore we can avoid too small or too large size

7 of communication messages. The message size would range

8 from a few KB to tens of MB. $W_{1}$ and $W_{2}$ are chosen in

$9[0,5]$. We determine loop tiling parameters $\left(P_{x 1}, P_{y 1}, U_{x 1}\right.$,

$10 U_{z 1}, P_{x 2}, P_{z 2}, U_{y 2}$, and $\left.U_{z 2}\right)$ in the way that the number of

11 elements of each sub-tile ranges in $\left[2^{10}, 2^{17}\right]$. Thus we can

12 utilize hardware caches with the size between a few KB not. So, we can also take into account the boundary values in the original parameter space after reducing the search space. For example, when $N_{x}=640$ and $D=32, T_{1}$ can be $1,2,4,8,16$, or 20 . As an exception, the log-scale reduction is not applied to $W_{1}, W_{2}, R_{y}, V$, and $S$ because there are few possible values for those parameters.

The fourth technique is called tile extrapolation. Our 3-D FFT algorithm repeats similar computation and communication on many tiles. So the execution time on tiles will be similar to one another. To reduce the execution time of each configuration, we execute the for-loops in Algorithm 1 only for the first few tiles. We then estimate the whole execution time by extrapolating the measured time. When the window size is not zero, we do not start extrapolation until the communication window is filled up. For example, if $W_{1}=2$, we start extrapolation at the third tile after the first two tiles fill up the communication window.

\section{Evaluation}

We first introduce our experimental platforms and three other approaches to compare with our approach. Second, we describe the results of small scale experiments. We will show how fast our 3-D FFT is compared to the others, and where the improvement comes from. Then we describe the results of large-scale experiments and show how well our approach scales as the number of cores increases. Last, we quantify the auto-tuning cost.

\subsection{Platforms and Comparison Models}

We use two platforms for our experiments. The first platform, which is named Edison, is a Cray XC30 machine at NERSC. Each node consists of two 12-core Intel Ivy Bridge processors (124,608 cores total in the machine). Each core has L1 and L2 caches, with $64 \mathrm{~KB}$ and $256 \mathrm{~KB}$, respectively. A 30MB L3 cache is shared between 12 cores on a processor. We used all 24 cores per node in our experiments. The Cray Aries with Dragonfly topology is used to connect nodes. For a non-blocking all-to-all operation on Edison, we used MPI_Ialltoall of the Cray Message Passing Toolkit 6.2.0 that is derived from the MPICH implementation. FFTW 3.3.0.4 is used. We used the Intel $\mathrm{C}$ and Fortran compilers and the Cray UPC compiler with -03 option to compile codes. The second platform is Hopper, a Cray XE6 machine at NERSC. Each node contains two 12-core AMD MagnyCours 2.1GHz processors $(153,216$ cores total in the machine). Each core has L1 and L2 caches, with $64 \mathrm{~KB}$ and $512 \mathrm{~KB}$, respectively. One $6 \mathrm{MB}$ L3 cache is shared between six cores on a processor. We used all 24 cores per node. Nodes are connected via a Cray Gemini Network that forms a 3-D torus. We used the Cray Message Passing Toolkit 6.0.1 and FFTW 3.3.0.4. We compiled codes using the PGI C and Fortran compilers and the Cray UPC compiler with -fast option. 
Table 2: Parallel 3-D FFT Time (seconds) and Parameter Values Found via Auto-Tuning (Small Scale)

(a) Edison

\begin{tabular}{|r|r||r||r|r|r|r|r|r|r|r|r|r|r|r|r|r|r|r|r|r|r|r|r|r|r|r|}
\hline$p$ & $N^{3}$ & time(s) & $D$ & $T_{1}$ & $W_{1}$ & $P_{x 1}$ & $P_{y 1}$ & $F_{z}$ & $F_{p 1}$ & $U_{x 1}$ & $U_{z 1}$ & $F_{u 1}$ & $F_{y 1}$ & $R_{y}$ & $T_{2}$ & $W_{2}$ & $P_{z 2}$ & $P_{x 2}$ & $F_{y 2}$ & $F_{p 2}$ & $U_{z 2}$ & $U_{y 2}$ & $F_{u 2}$ & $F_{x}$ & $V$ & $S$ \\
\hline \hline 256 & $768^{3}$ & 0.195 & 1 & 32 & 5 & 1 & 1 & 64 & 32 & 1 & 2 & 32 & 64 & $\mathrm{X}$ & $\mathrm{X}$ & $\mathrm{X}$ & $\mathrm{X}$ & $\mathrm{X}$ & $\mathrm{X}$ & $\mathrm{X}$ & $\mathrm{X}$ & $\mathrm{X}$ & $\mathrm{X}$ & $\mathrm{X}$ & 0 & 1 \\
256 & $1024^{3}$ & 0.516 & 1 & 256 & 1 & 4 & 4 & 256 & 16 & 2 & 4 & 16 & 1024 & $\mathrm{X}$ & $\mathrm{X}$ & $\mathrm{X}$ & $\mathrm{X}$ & $\mathrm{X}$ & $\mathrm{X}$ & $\mathrm{X}$ & $\mathrm{X}$ & $\mathrm{X}$ & $\mathrm{X}$ & $\mathrm{X}$ & 0 & 1 \\
256 & $1280^{3}$ & 0.979 & 1 & 8 & 1 & 2 & 2 & 32 & 8 & 1 & 1 & 32 & 32 & $\mathrm{X}$ & $\mathrm{X}$ & $\mathrm{X}$ & $\mathrm{X}$ & $\mathrm{X}$ & $\mathrm{X}$ & $\mathrm{X}$ & $\mathrm{X}$ & $\mathrm{X}$ & $\mathrm{X}$ & $\mathrm{X}$ & 0 & 1 \\
256 & $1536^{3}$ & 1.909 & 1 & 128 & 4 & 8 & 2 & 256 & 32 & 1 & 2 & 128 & 128 & $\mathrm{X}$ & $\mathrm{X}$ & $\mathrm{X}$ & $\mathrm{X}$ & $\mathrm{X}$ & $\mathrm{X}$ & $\mathrm{X}$ & $\mathrm{X}$ & $\mathrm{X}$ & $\mathrm{X}$ & $\mathrm{X}$ & 0 & 1 \\
256 & $1792^{3}$ & 2.898 & 1 & 128 & 4 & 1 & 1 & 256 & 128 & 1 & 4 & 32 & 512 & $\mathrm{X}$ & $\mathrm{X}$ & $\mathrm{X}$ & $\mathrm{X}$ & $\mathrm{X}$ & $\mathrm{X}$ & $\mathrm{X}$ & $\mathrm{X}$ & $\mathrm{X}$ & $\mathrm{X}$ & $\mathrm{X}$ & 0 & 1 \\
256 & $2048^{3}$ & 4.749 & 256 & $\mathrm{X}$ & $\mathrm{X}$ & $\mathrm{X}$ & $\mathrm{X}$ & $\mathrm{X}$ & $\mathrm{X}$ & $\mathrm{X}$ & $\mathrm{X}$ & $\mathrm{X}$ & $\mathrm{X}$ & $\mathrm{X}$ & 128 & 2 & 4 & 8 & 64 & 32 & 8 & 1 & 32 & 128 & 0 & 0 \\
\hline 512 & $768^{3}$ & 0.113 & 128 & 1 & 2 & 1 & 32 & 2 & 1 & 1 & 8 & 2 & 8 & 5 & 8 & 1 & 8 & 4 & 16 & 1 & 2 & 1 & 8 & 32 & 0 & 0 \\
512 & $1024^{3}$ & 0.298 & 32 & 4 & 3 & 1 & 8 & 32 & 8 & 2 & 2 & 2 & 32 & 5 & 8 & 3 & 2 & 4 & 64 & 8 & 2 & 4 & 8 & 32 & 0 & 0 \\
512 & $1280^{3}$ & 0.492 & 32 & 2 & 4 & 1 & 8 & 16 & 2 & 1 & 32 & 2 & 8 & 4 & 4 & 2 & 2 & 2 & 32 & 8 & 1 & 8 & 8 & 32 & 0 & 0 \\
512 & $1536^{3}$ & 1.017 & 1 & 512 & 2 & 2 & 1 & 512 & 64 & 1 & 2 & 64 & 1024 & $\mathrm{X}$ & $\mathrm{X}$ & $\mathrm{X}$ & $\mathrm{X}$ & $\mathrm{X}$ & $\mathrm{X}$ & $\mathrm{X}$ & $\mathrm{X}$ & $\mathrm{X}$ & $\mathrm{X}$ & $\mathrm{X}$ & 0 & 1 \\
512 & $1792^{3}$ & 1.665 & 1 & 256 & 1 & 1 & 1 & 512 & 512 & 2 & 1 & 512 & 64 & $\mathrm{X}$ & $\mathrm{X}$ & $\mathrm{X}$ & $\mathrm{X}$ & $\mathrm{X}$ & $\mathrm{X}$ & $\mathrm{X}$ & $\mathrm{X}$ & $\mathrm{X}$ & $\mathrm{X}$ & $\mathrm{X}$ & 2 & 1 \\
512 & $2048^{3}$ & 2.277 & 1 & 256 & 2 & 1 & 2 & 256 & 128 & 2 & 2 & 64 & 512 & $\mathrm{X}$ & $\mathrm{X}$ & $\mathrm{X}$ & $\mathrm{X}$ & $\mathrm{X}$ & $\mathrm{X}$ & $\mathrm{X}$ & $\mathrm{X}$ & $\mathrm{X}$ & $\mathrm{X}$ & $\mathrm{X}$ & 0 & 0 \\
\hline
\end{tabular}

(b) Hopper

\begin{tabular}{|r|r||r||r|r|r|r|r|r|r|r|r|r|r|r|r|r|r|r|r|r|r|r|r|r|r|r|}
\hline$p$ & $N^{3}$ & time $(\mathrm{s})$ & $D$ & $T_{1}$ & $W_{1}$ & $P_{x 1}$ & $P_{y 1}$ & $F_{z}$ & $F_{p 1}$ & $U_{x 1}$ & $U_{z 1}$ & $F_{u 1}$ & $F_{y 1}$ & $R_{y}$ & $T_{2}$ & $W_{2}$ & $P_{z 2} 2$ & $P_{x 2}$ & $F_{y 2}$ & $F_{p 2}$ & $U_{z 2}$ & $U_{y 2}$ & $F_{u 2}$ & $F_{x}$ & $V$ & $S$ \\
\hline \hline 256 & $768^{3}$ & 0.450 & 32 & 4 & 2 & 1 & 4 & 8 & 4 & 2 & 4 & 4 & 16 & 4 & 4 & 2 & 2 & 4 & 32 & 4 & 2 & 2 & 8 & 8 & 0 & 0 \\
256 & $1024^{3}$ & 1.077 & 8 & 8 & 2 & 2 & 4 & 16 & 16 & 2 & 4 & 8 & 32 & 7 & 4 & 2 & 1 & 16 & 8 & 4 & 2 & 2 & 8 & 32 & 0 & 0 \\
256 & $1280^{3}$ & 2.155 & 16 & 8 & 2 & 2 & 8 & 8 & 2 & 1 & 1 & 8 & 128 & 9 & 2 & 1 & 2 & 4 & 2 & 8 & 1 & 16 & 2 & 32 & 0 & 0 \\
256 & $1536^{3}$ & 3.726 & 1 & 128 & 5 & 2 & 1 & 64 & 128 & 4 & 4 & 32 & 512 & $\mathrm{X}$ & $\mathrm{X}$ & $\mathrm{X}$ & $\mathrm{X}$ & $\mathrm{X}$ & $\mathrm{X}$ & $\mathrm{X}$ & $\mathrm{X}$ & $\mathrm{X}$ & $\mathrm{X}$ & $\mathrm{X}$ & 0 & 0 \\
256 & $1792^{3}$ & 5.733 & 1 & 128 & 1 & 4 & 4 & 128 & 16 & 4 & 2 & 32 & 256 & $\mathrm{X}$ & $\mathrm{X}$ & $\mathrm{X}$ & $\mathrm{X}$ & $\mathrm{X}$ & $\mathrm{X}$ & $\mathrm{X}$ & $\mathrm{X}$ & $\mathrm{X}$ & $\mathrm{X}$ & $\mathrm{X}$ & 0 & 0 \\
256 & $2048^{3}$ & 8.691 & 256 & $\mathrm{X}$ & $\mathrm{X}$ & $\mathrm{X}$ & $\mathrm{X}$ & $\mathrm{X}$ & $\mathrm{X}$ & $\mathrm{X}$ & $\mathrm{X}$ & $\mathrm{X}$ & $\mathrm{X}$ & $\mathrm{X}$ & 128 & 1 & 1 & 4 & 128 & 128 & 8 & 4 & 32 & 512 & 0 & 0 \\
\hline 512 & $768^{3}$ & 0.277 & 32 & 8 & 2 & 2 & 16 & 8 & 4 & 4 & 2 & 4 & 8 & 3 & 8 & 1 & 1 & 2 & 32 & 4 & 4 & 4 & 8 & 64 & 0 & 0 \\
512 & $1024^{3}$ & 0.567 & 32 & 4 & 2 & 2 & 8 & 16 & 8 & 2 & 16 & 4 & 8 & 5 & 2 & 2 & 2 & 4 & 16 & 8 & 1 & 8 & 4 & 8 & 0 & 0 \\
512 & $1280^{3}$ & 1.232 & 64 & 4 & 2 & 1 & 8 & 16 & 8 & 2 & 8 & 8 & 16 & 4 & 16 & 2 & 2 & 8 & 64 & 16 & 2 & 8 & 16 & 128 & 0 & 0 \\
512 & $1536^{3}$ & 2.060 & 32 & 4 & 2 & 2 & 2 & 32 & 4 & 4 & 8 & 4 & 16 & 1 & 4 & 1 & 2 & 4 & 64 & 16 & 2 & 2 & 4 & 64 & 0 & 0 \\
512 & $1792^{3}$ & 3.791 & 32 & 4 & 2 & 2 & 4 & 16 & 8 & 2 & 4 & 16 & 32 & 3 & 4 & 1 & 2 & 4 & 16 & 8 & 4 & 4 & 4 & 16 & 0 & 0 \\
512 & $2048^{3}$ & 5.251 & 128 & 2 & 1 & 1 & 8 & 16 & 8 & 1 & 8 & 4 & 16 & 0 & 64 & 2 & 2 & 4 & 512 & 32 & 1 & 4 & 128 & 64 & 0 & 0 \\
\hline
\end{tabular}

We compare four different methods for parallel 3-D FFT. FFTW is the FFTW library [9] that uses 1-D decomposition and blocking MPI communication. We tune and optimize the parallel 3-D FFT computation of FFTW by using the FFTW_MEASURE option. Second is DCMP, which is the 2DECOMP\&FFT library [5] that uses 2-D decomposition and blocking MPI collective communication. 3 For fair comparison with our tuned approach, we exhaustively run all the possible process grids of $p_{1} \times p_{2}$ and choose the option with the best performance. Third, we compare our approach with UPCF, which is a UPC code [8] that is based on 2-D decomposition. UPCF overlaps computation with communication through one-sided point-to-point communication operations. We downloaded the UPC implementation of the NAS Parallel Benchmark FT (UPC-FT) from the NERSC website. Then we built UPCF by extracting a core part of UPC-FT containing the 3-D FFT computation. Like DCMP, UPCF tunes $p_{1} \times p_{2}$ exhaustively. Last is NEW, which is our method described in Section 3. NEW is auto-tuned by the Nelder-

${ }^{3}$ We do not use P3DFFT [4] in experiments because it does not support the complex-to-complex transform. P3DFFT is reported to show the similar performance to 2DECOMP\&FFT for the real-to-complex transform. [17]
Mead strategy of Active Harmony as described in Section 4 Since DCMP, UPCF, and NEW rely on the FFTW library for 1-D FFT computation, we tune those computations with the FFTW_MEASURE option.

\subsection{Small Scale Experiments}

We first performed experiments on a relatively small number (256 and 512) of compute cores. Input sizes are $768^{3}$ to $2048^{3}$ complex numbers, which are sufficiently large so that we can run FFTW and use 1-D decomposition.

\subsubsection{Parallel 3-D FFT Performance}

The third column of Table 2(a) shows the 3-D FFT execution time of our NEW approach on Edison. Figure 5(a) shows the speedup of NEW over other approaches. $p$ is the number of cores used. $N$ is the number of elements on each dimension in a $3-\mathrm{D}$ input array. For each of the four algorithms being compared, we conducted five runs of tuning on different sets of computing nodes. To cope with the execution noise, we executed five runs of 3-D FFT with each of five tuning results, then picked the best performance out of the 25 runs. Figure $5(\mathrm{a})$ shows that NEW is faster than FFTW, DCMP, and UPCF for all different 


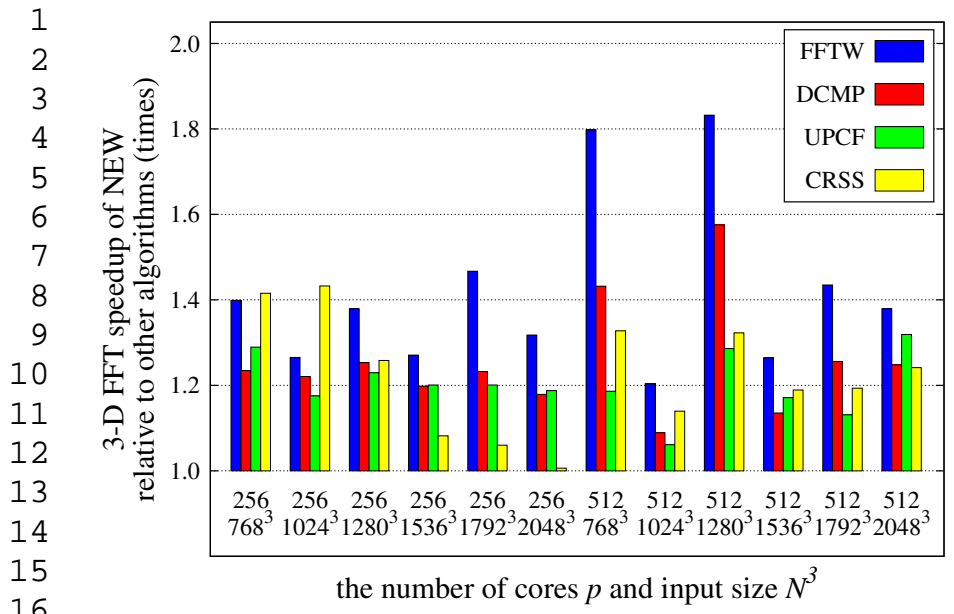

(a) Edison

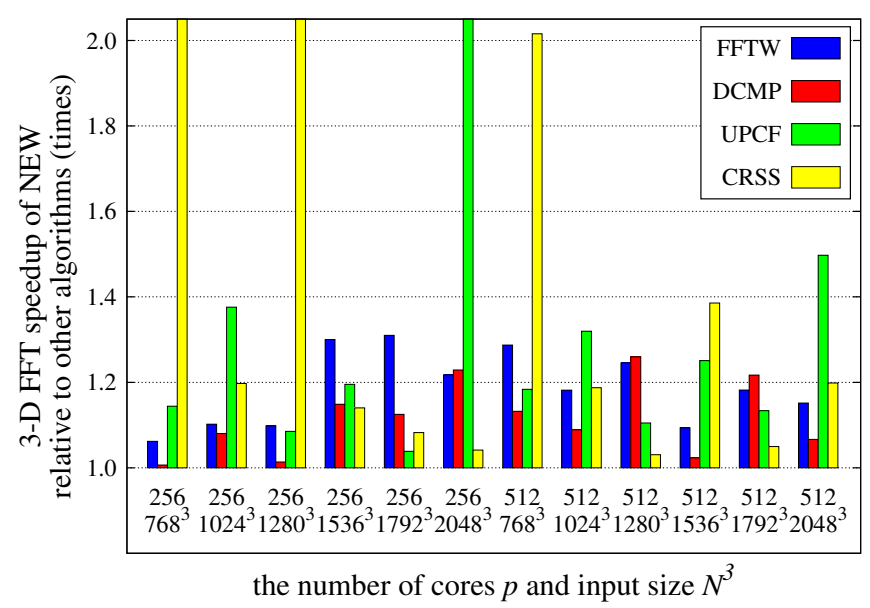

(b) Hopper settings of $p$ and $N$. NEW has speedup of up to $1.83 \times$ over FFTW, $1.58 \times$ over DCMP, and $1.32 \times$ over UPCF.

To better explain the effectiveness of NEW, we break down the performance of NEW for the $p=512$ and $N^{3}=$ $1024^{3}$ configuration in Figure 9(a) NEW-0 is a nonoverlapped version of NEW where $W_{1}, W_{2}$, and all the frequency parameters are set to be zero with all the other parameters equal to NEW. Also, lines 4 and 5 (also lines 11 and 12) in Algorithm 1 are replaced with MPI_Ialltoall and MPI_Wait on tile $i$. In Phase1, the all-to-all communication time (the MPI_Wait time marked with Wait1) of NEW-0 is 0.028 seconds on Edison, and NEW reduces the Wait1 time down to zero. In Phase2, the communication time (Wait2) of NEW-0 is 0.173 seconds. The Wait2 time of NEW is reduced to only 0.048 seconds as the "overlappable" computation time (FFTy2+FFTx and Pack2+Unpack2) of NEW is 0.125 seconds. So the sum of computation time and communication time of NEW is equal to the communication time of NEW-0. The reduced communication time (Wait1 and Wait2) of NEW represents NEW nearly achieves the perfect computationcommunication overlap on Edison. The overhead for the computation-communication overlap is quantified as the time for calling MPI_Test. NEW spends 0.029 seconds for MPI_Test (Test1 and Test2) on Edison. Since this overhead is much shorter than the reduced communication time (Wait1 and Wait2) of NEW (0.153 seconds), NEW could successfully improve the overall FFT performance from NEW-0.

The high degree of overlap explains why NEW is faster than the other approaches. Since FFTW and DCMP do not exploit non-blocking communication, the performance is lower than NEW as shown in Figure 5(a) UPCF over- laps computation and communication, but it is still slower than NEW. There are three possible reasons of UPCF's low performance. First, the UPCF design [8] misses some computation (Unpack1, Unpack2, and FFTx) to overlap with communication. Second, UPCF is not able to tune the size of communication message as there is no concept of $T_{1}$ and $T_{2}$. Last, UPCF uses the point-to-point communication while NEW is based on the optimized collective operation. Note that Wait1 of NEW-0 is shorter than Wait2 in Figure 9(a). The reason of the short communication time of Phase1 is fast intra-node communication. We map MPI processes to a 3-D array along the $y$ dimension first as shown in Figure 2 By default, Edison uses the SMP-style mapping between MPI processes and physical cores, so it fills up one node before going to next. Thus Phase1 involves a large amount of intra-node communication, which should be faster than inter-node communication of Phase2.

We conducted the same experiment on Hopper as what we did on Edison. Table 2(b) shows the 3-D FFT execution time on Hopper. Figure 5(b) shows the speedup of NEW over the other approaches. 4 NEW is still the fastest on Hopper (up to $1.31 \times \mathrm{FFTW}, 1.26 \times \mathrm{DCMP}$, and $1.50 \times \mathrm{UPCF}$ ), but the speedup is somewhat lower than the speedup on Edison. This low speedup on Hopper comes from the non-maximized overlap. In Figure 9(a) NEW on Hopper takes around 0.070 seconds for Phase2 communication (marked with Wait2), which means a nonoptimized overlap. NEW is supposed to have zero communication time as the computation time (FFTy2+FFTx and

${ }^{4} \mathrm{UPCF}$ crashes at $p=256$ and $N^{3}=2048^{3}$ on Hopper with an out-of-memory error. UPCF requires a large amount of memory as it only supports the out-of-place transform. 
(a) Edison (Strong Scaling)

\begin{tabular}{|r|r||r||r|r|r|r|r|r|r|r|r|r|r|r|r|r|r|r|r|r|r|r|r|r|r|r|}
\hline$p$ & $N^{3}$ & time $(\mathrm{s})$ & $D$ & $T_{1}$ & $W_{1}$ & $P_{x 1}$ & $P_{y 1}$ & $F_{z}$ & $F_{p 1}$ & $U_{x 1}$ & $U_{z 1}$ & $F_{u 1}$ & $F_{y 1}$ & $R_{y}$ & $T_{2}$ & $W_{2}$ & $P_{z 2}$ & $P_{x 2}$ & $F_{y 2}$ & $F_{p 2}$ & $U_{z 2}$ & $U_{y 2}$ & $F_{u 2}$ & $F_{x}$ & $V$ & $S$ \\
\hline \hline 128 & $1024^{3}$ & 0.965 & 4 & 4 & 3 & 1 & 8 & 32 & 8 & 1 & 4 & 8 & 16 & 8 & 8 & 1 & 4 & 16 & 4 & 32 & 2 & 4 & 32 & 16 & 0 & 0 \\
256 & $1024^{3}$ & 0.516 & 1 & 256 & 1 & 4 & 4 & 256 & 16 & 2 & 4 & 16 & 1024 & $\mathrm{X}$ & $\mathrm{X}$ & $\mathrm{X}$ & $\mathrm{X}$ & $\mathrm{X}$ & $\mathrm{X}$ & $\mathrm{X}$ & $\mathrm{X}$ & $\mathrm{X}$ & $\mathrm{X}$ & $\mathrm{X}$ & 0 & 1 \\
512 & $1024^{3}$ & 0.298 & 32 & 4 & 3 & 1 & 8 & 32 & 8 & 2 & 2 & 2 & 32 & 5 & 8 & 3 & 2 & 4 & 64 & 8 & 2 & 4 & 8 & 32 & 0 & 0 \\
1024 & $1024^{3}$ & 0.159 & 64 & 4 & 2 & 2 & 32 & 4 & 2 & 2 & 2 & 4 & 8 & 5 & 16 & 1 & 4 & 8 & 32 & 8 & 4 & 4 & 8 & 32 & 0 & 0 \\
2048 & $1024^{3}$ & 0.100 & 256 & 2 & 2 & 1 & 16 & 4 & 4 & 1 & 32 & 2 & 64 & 5 & 16 & 4 & 4 & 2 & 32 & 16 & 8 & 2 & 4 & 32 & 0 & 0 \\
4096 & $1024^{3}$ & 0.055 & 256 & 4 & 0 & 1 & 4 & 0 & 0 & 2 & 4 & 0 & 0 & 3 & 16 & 2 & 8 & 4 & 64 & 4 & 2 & 4 & 8 & 64 & 0 & 0 \\
8192 & $1024^{3}$ & 0.030 & 512 & 2 & 0 & 2 & 8 & 0 & 0 & 1 & 8 & 0 & 0 & 2 & 32 & 2 & 8 & 1 & 32 & 4 & 4 & 1 & 8 & 32 & 0 & 0 \\
16384 & $1024^{3}$ & 0.018 & 256 & 4 & 0 & 2 & 16 & 0 & 0 & 2 & 4 & 0 & 0 & 3 & 8 & 2 & 2 & 4 & 16 & 8 & 4 & 4 & 2 & 32 & 0 & 0 \\
32768 & $1024^{3}$ & 0.012 & 256 & 4 & 0 & 2 & 4 & 0 & 0 & 4 & 4 & 0 & 0 & 5 & 8 & 0 & 4 & 4 & 0 & 0 & 4 & 1 & 0 & 0 & 0 & 0 \\
\hline
\end{tabular}

(b) Hopper (Strong Scaling)

\begin{tabular}{|r|r||r||r|r|r|r|r|r|r|r|r|r|r|r|r|r|r|r|r|r|r|r|r|r|r|r|}
\hline$p$ & $N^{3}$ & time(s) & $D$ & $T_{1}$ & $W_{1}$ & $P_{x 1}$ & $P_{y 1}$ & $F_{z}$ & $F_{p 1}$ & $U_{x 1}$ & $U_{z 1}$ & $F_{u 1}$ & $F_{y 1}$ & $R_{y}$ & $T_{2}$ & $W_{2}$ & $P_{z 2}$ & $P_{x 2}$ & $F_{y 2}$ & $F_{p 2}$ & $U_{z 2}$ & $U_{y 2}$ & $F_{u 2}$ & $F_{x}$ & $V$ & $S$ \\
\hline \hline 128 & $1024^{3}$ & 1.673 & 128 & $\mathrm{X}$ & $\mathrm{X}$ & $\mathrm{X}$ & $\mathrm{X}$ & $\mathrm{X}$ & $\mathrm{X}$ & $\mathrm{X}$ & $\mathrm{X}$ & $\mathrm{X}$ & $\mathrm{X}$ & $\mathrm{X}$ & 64 & 5 & 2 & 8 & 64 & 32 & 8 & 2 & 16 & 128 & 0 & 0 \\
256 & $1024^{3}$ & 1.077 & 8 & 8 & 2 & 2 & 4 & 16 & 16 & 2 & 4 & 8 & 32 & 7 & 4 & 2 & 1 & 16 & 8 & 4 & 2 & 2 & 8 & 32 & 0 & 0 \\
512 & $1024^{3}$ & 0.567 & 32 & 4 & 2 & 2 & 8 & 16 & 8 & 2 & 16 & 4 & 8 & 5 & 2 & 2 & 2 & 4 & 16 & 8 & 1 & 8 & 4 & 8 & 0 & 0 \\
1024 & $1024^{3}$ & 0.292 & 64 & 4 & 2 & 1 & 8 & 16 & 8 & 1 & 8 & 8 & 16 & 4 & 8 & 4 & 2 & 8 & 64 & 8 & 2 & 4 & 4 & 32 & 0 & 0 \\
2048 & $1024^{3}$ & 0.272 & 64 & 4 & 1 & 2 & 4 & 64 & 8 & 4 & 2 & 8 & 8 & 3 & 4 & 3 & 1 & 2 & 32 & 16 & 2 & 4 & 8 & 16 & 0 & 0 \\
4096 & $1024^{3}$ & 0.167 & 16 & 32 & 0 & 2 & 2 & 0 & 0 & 8 & 4 & 0 & 0 & 1 & 4 & 0 & 2 & 32 & 0 & 0 & 4 & 4 & 0 & 0 & 0 & 1 \\
8192 & $1024^{3}$ & 0.125 & 8 & 128 & 0 & 32 & 1 & 0 & 0 & 2 & 1 & 0 & 0 & 8 & 1 & 0 & 1 & 64 & 0 & 0 & 1 & 16 & 0 & 0 & 0 & 0 \\
16384 & $1024^{3}$ & 0.062 & 64 & 16 & 0 & 2 & 2 & 0 & 0 & 8 & 2 & 0 & 0 & 4 & 4 & 0 & 1 & 4 & 0 & 0 & 2 & 2 & 0 & 0 & 0 & 0 \\
32768 & $1024^{3}$ & 0.049 & 128 & 8 & 0 & 4 & 2 & 0 & 0 & 2 & 2 & 0 & 0 & 3 & 4 & 0 & 2 & 4 & 0 & 0 & 2 & 2 & 0 & 0 & 0 & 1 \\
\hline
\end{tabular}

(c) Edison (Weak Scaling)

\begin{tabular}{|r|r|r|r||r||r|r|r|r|r|r|r|r|r|r|r|r|r|r|r|r|r|r|r|r|r|r|r|r|}
\hline$p$ & $N_{x}$ & $N_{y}$ & $N_{z}$ & time $(\mathrm{s})$ & $D$ & $T_{1}$ & $W_{1}$ & $P_{x 1}$ & $P_{y 1}$ & $F_{z}$ & $F_{p 1}$ & $U_{x 1}$ & $U_{z 1}$ & $F_{u 1}$ & $F_{y 1}$ & $R_{y}$ & $T_{2}$ & $W_{2}$ & $P_{z 2}$ & $P_{x 2}$ & $F_{y 2}$ & $F_{p 2}$ & $U_{z 2}$ & $U_{y 2}$ & $F_{u 2}$ & $F_{x}$ & $V$ & $S$ \\
\hline \hline 128 & 512 & 512 & 512 & 0.114 & 1 & 8 & 4 & 1 & 2 & 32 & 16 & 2 & 1 & 16 & 16 & $\mathrm{X}$ & $\mathrm{X}$ & $\mathrm{X}$ & $\mathrm{X}$ & $\mathrm{X}$ & $\mathrm{X}$ & $\mathrm{X}$ & $\mathrm{X}$ & $\mathrm{X}$ & $\mathrm{X}$ & $\mathrm{X}$ & 0 & 1 \\
256 & 512 & 512 & 1024 & 0.139 & 1 & 32 & 5 & 1 & 2 & 32 & 32 & 1 & 2 & 32 & 32 & $\mathrm{X}$ & $\mathrm{X}$ & $\mathrm{X}$ & $\mathrm{X}$ & $\mathrm{X}$ & $\mathrm{X}$ & $\mathrm{X}$ & $\mathrm{X}$ & $\mathrm{X}$ & $\mathrm{X}$ & $\mathrm{X}$ & 0 & 1 \\
512 & 1024 & 1024 & 512 & 0.155 & 1 & 64 & 4 & 32 & 1 & 128 & 4 & 2 & 1 & 32 & 64 & $\mathrm{X}$ & $\mathrm{X}$ & $\mathrm{X}$ & $\mathrm{X}$ & $\mathrm{X}$ & $\mathrm{X}$ & $\mathrm{X}$ & $\mathrm{X}$ & $\mathrm{X}$ & $\mathrm{X}$ & $\mathrm{X}$ & 0 & 1 \\
1024 & 1024 & 1024 & 1024 & 0.159 & 64 & 4 & 2 & 2 & 32 & 4 & 2 & 2 & 2 & 4 & 8 & 5 & 16 & 1 & 4 & 8 & 32 & 8 & 4 & 4 & 8 & 32 & 0 & 0 \\
2048 & 1024 & 1024 & 2048 & 0.190 & 128 & 1 & 4 & 1 & 64 & 4 & 1 & 1 & 64 & 2 & 16 & 3 & 64 & 1 & 8 & 8 & 256 & 8 & 2 & 8 & 16 & 32 & 0 & 0 \\
4096 & 2048 & 2048 & 1024 & 0.202 & 256 & 2 & 1 & 1 & 128 & 16 & 2 & 2 & 1 & 8 & 128 & 7 & 4 & 4 & 2 & 2 & 32 & 8 & 1 & 4 & 8 & 32 & 0 & 0 \\
8192 & 2048 & 2048 & 2048 & 0.207 & 512 & 2 & 1 & 1 & 16 & 64 & 4 & 1 & 4 & 4 & 16 & 1 & 16 & 2 & 8 & 2 & 64 & 8 & 4 & 2 & 16 & 64 & 0 & 0 \\
16384 & 2048 & 2048 & 4096 & 0.288 & 1024 & 2 & 0 & 1 & 32 & 0 & 0 & 2 & 1 & 0 & 0 & 2 & 64 & 3 & 1 & 1 & 128 & 128 & 4 & 2 & 16 & 128 & 0 & 0 \\
32768 & 4096 & 4096 & 2048 & 0.341 & 256 & 4 & 2 & 1 & 4 & 64 & 32 & 4 & 8 & 2 & 32 & 9 & 8 & 1 & 2 & 8 & 64 & 8 & 1 & 16 & 8 & 64 & 0 & 0 \\
\hline
\end{tabular}

(d) Hopper (Weak Scaling)

\begin{tabular}{|c|c|c|c|c|c|c|c|c|c|c|c|c|c|c|c|c|c|c|c|c|c|c|c|c|c|c|c|}
\hline$p$ & $N_{x}$ & $N_{y}$ & $N_{z}$ & & $D$ & $T_{1}$ & & 1 & $P_{y 1}$ & $F_{z}$ & $E$ & 1 & L & $F_{u 1}$ & $F_{y}$ & \begin{tabular}{|l|l}
$R_{y}$ & \\
\end{tabular} & $T_{2}$ & $W$ & $T$ & $P_{x 2}$ & $F_{y}$ & $F_{p 2}$ & $J_{z 2}$ & $U_{y 2}$ & $F_{u 2}$ & $F_{x}$ & \\
\hline 128 & $\overline{512}$ & $\overline{512}$ & 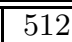 & $\overline{13 \mid}$ & 1 & 128 & 1 & $\overline{2}$ & 2 & 256 & ב32 & 8 & $\overline{1}$ & \begin{tabular}{|c|}
64 \\
\end{tabular} & 512 & 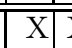 & & $\overline{\mathrm{X}}$ & $\overline{\mathrm{X}}$ & $\overline{\mathrm{X}}$ & $\overline{\mathrm{X}}$ & $\overline{\mathrm{X}}$ & $\overline{\mathrm{X}}$ & $\overline{\mathrm{X}}$ & $\overline{\bar{X}}$ & $\overline{\overline{\mathrm{X}}}$ & 0 \\
\hline 2 & 512 & 512 & & & 16 & 8 & 1 & & 32 & (4) & & & & & 64 & & 2 & 4 & 1 & 16 & & & & & & & \\
\hline 512 & 24 & 1024 & & & 64 & 4 & 2 & & 32 & 64 & & & & & 16 & & & & 2 & 16 & 16 & & & 2 & 16 & 128 & \\
\hline & & & & & 6 & & 2 & & & 1 & & & & & 1 & & & & ? & 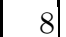 & 64 & & & & & 32 & \\
\hline & & & & & & & & & & 32 & & & & & & 3 & & & & & & & & & & & \\
\hline & & & & & 2 & & & & & 16 & & & & & 1 & 1 & & o & & & & & 2 & & & & \\
\hline & & & & & 512 & 4 & & 2 & 32 & & & & 16 & & 128 & 1 & & 0 & & 2 & 0 & & 16 & & & 0 & \\
\hline & & 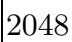 & & 996 & 1024 & 2 & 0 & & & 0 & & & & 0 & & 26 & 6 & & & & 128 & 32 & 2 & 2 & 64 & \begin{tabular}{|l|l|}
4 & 128
\end{tabular} & \\
\hline 768 & 96 & 96 & 20 & 1.1 & 12 & & 2 & & & 32 & & & & & 32 & 1 & 16 & & & & 64 & 321 & & & & & \\
\hline
\end{tabular}


1

2

3

4

5

6

7

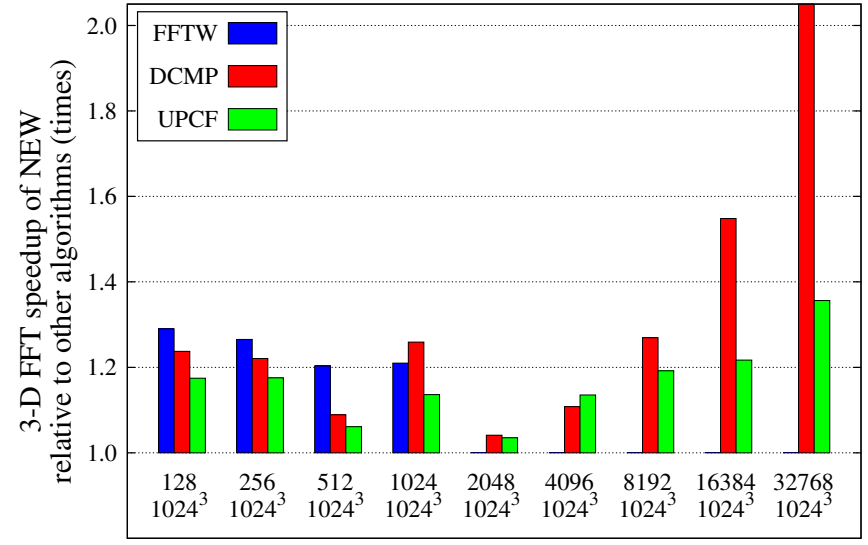

the number of cores $p$ and input size $N^{3}$

(a) Edison (Strong Scaling)

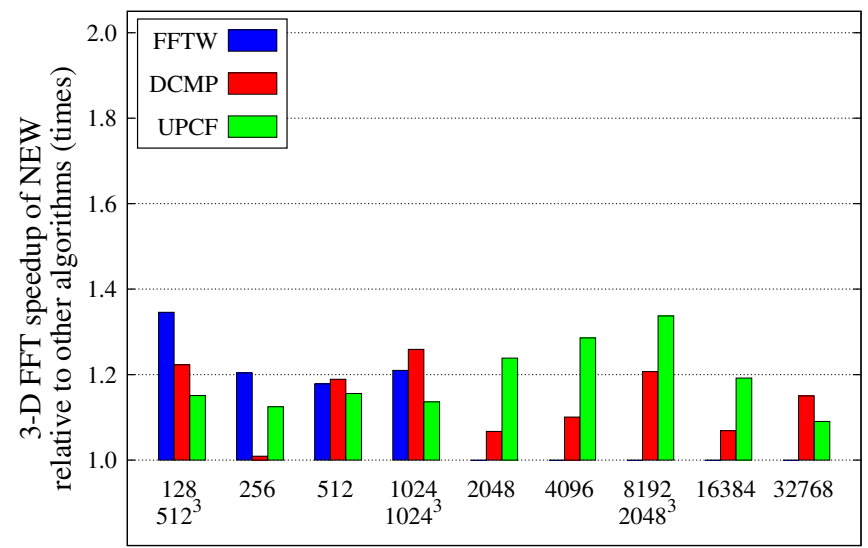

the number of cores $p$ and input size $N^{3}$

(c) Edison (Weak Scaling)

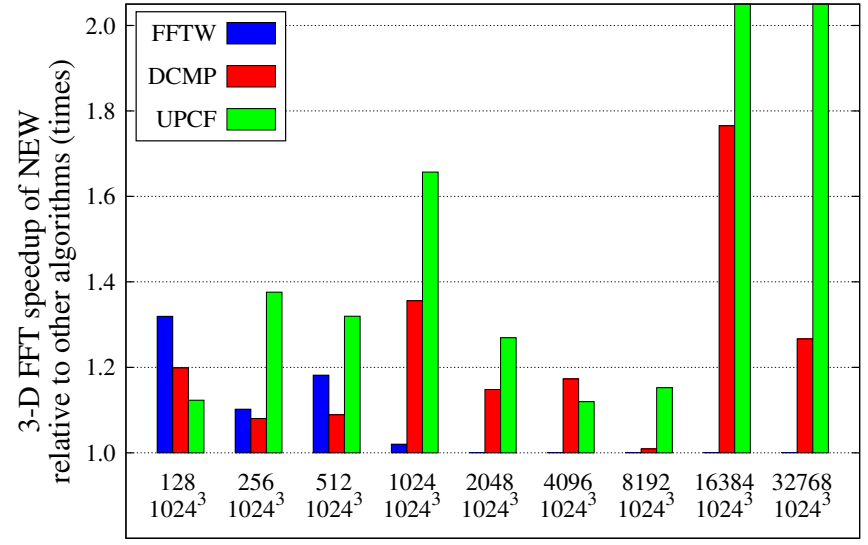

the number of cores $p$ and input size $N^{3}$

(b) Hopper (Strong Scaling)

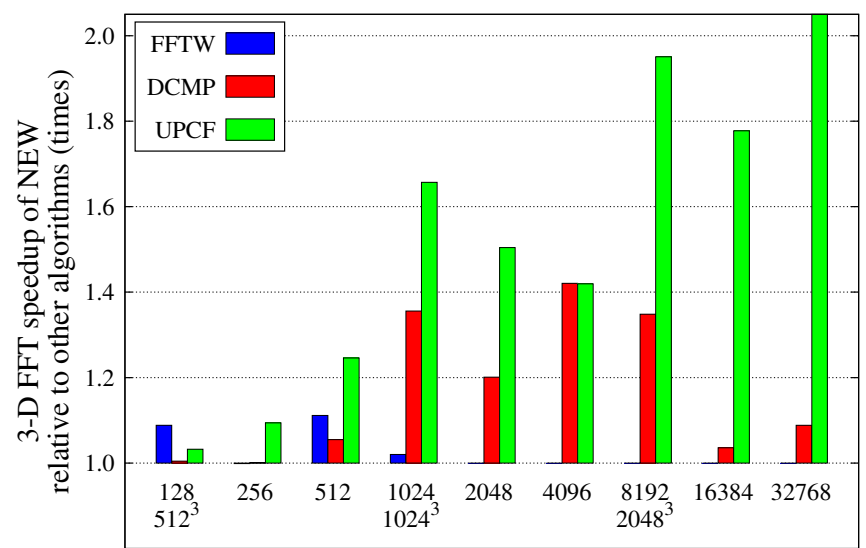

the number of cores $p$ and input size $N^{3}$

(d) Hopper (Weak Scaling)

Figure 6: Parallel 3-D FFT Speedup of NEW Relative to Other Algorithms on Large Scale Experiments: We used all 24 cores per node of Edison and Hopper. Strong scaling fixes an input size at $1024^{3}$ elements. Weak scaling doubles an input size from $512^{3}$ through $4096 \times 4096 \times 2048$. NEW shows speedup of up to $1.35 \times$ over FFTW, $2.57 \times$ over DCMP, and $1.36 \times$ over UPCF on Edison. The speedup on Hopper is up to $1.32 \times$ over FFTW, $1.77 \times$ over DCMP, and $1.95 \times$ over UPCF.

Pack2+Unpack2) of NEW is longer than communication time (Wait2) of NEW-0. We conjecture that the reason of non-optimized overlap on Hopper comes from memory access contention. Since we use all 24 cores per node, the communication of each core makes slow progress as it contends with memory access operations of other cores. We have a better overlap on Edison because Edison is a newer machine with higher memory bandwidth and larger L3 caches.

\subsubsection{The Necessity of Auto-Tuning}

Table 2 contains the auto-tuned parameter configurations of NEW. We can see that the best parameter values vary depending on system settings such as platform, input size, and the number of cores. Figure 10(a) shows the history of an auto-tuning execution for the setting of $p=512$ and $N^{3}=1024^{3}$. The $x$-axis means the tuning step number. The $y$-axis means the 3 -D FFT time for the parameter configuration found at each tuning step. The execution time decreases as the tuning proceeds. There is around $10 \times$ performance gap between the best and the worst configurations. Thus we should tune the parameters properly to achieve high performance of 3-D FFT.

We conducted cross-platform tests to further explore the necessity of auto-tuning, We executed the 3-D FFT code on Edison with a cross-platform parameter setting that uses the tuning result from Hopper (Table 2(b)]. CRSS in Figure 5(a) shows the speedup of the original setting over this cross-platform setting. The original setting is always faster (by up to $1.43 \times$ ) than the crossplatform settings. Likewise, we have executed the $3-\mathrm{D}$ FFT code on Hopper with the tuning result of Edison as shown in Table 2(a). CRSS in Figure 5(b) shows the speedup of NEW (up to $2.54 \times$ ) on Hopper relative to the cross-platform setting from Edison. The best configura- 


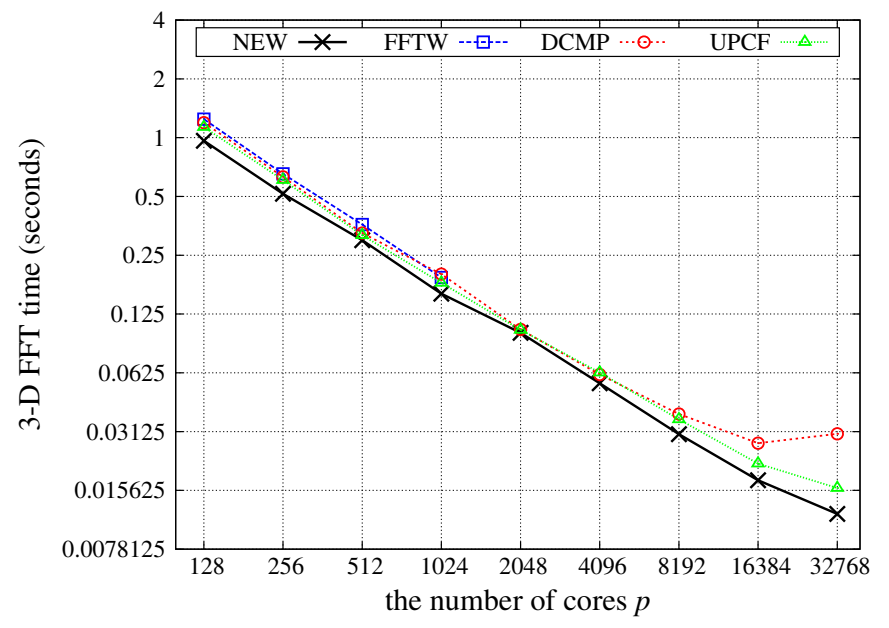

(a) Edison (Strong Scaling)

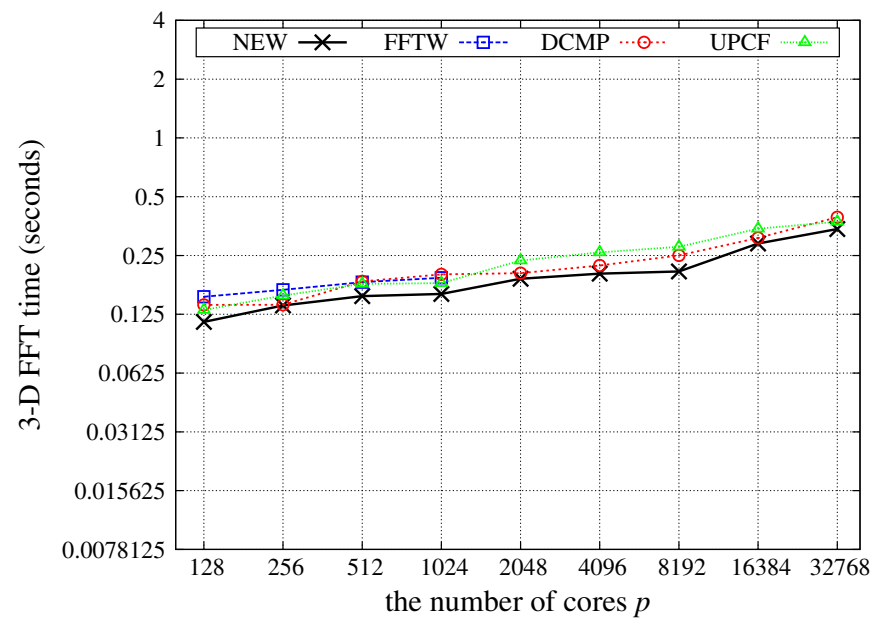

(c) Edison (Weak Scaling)

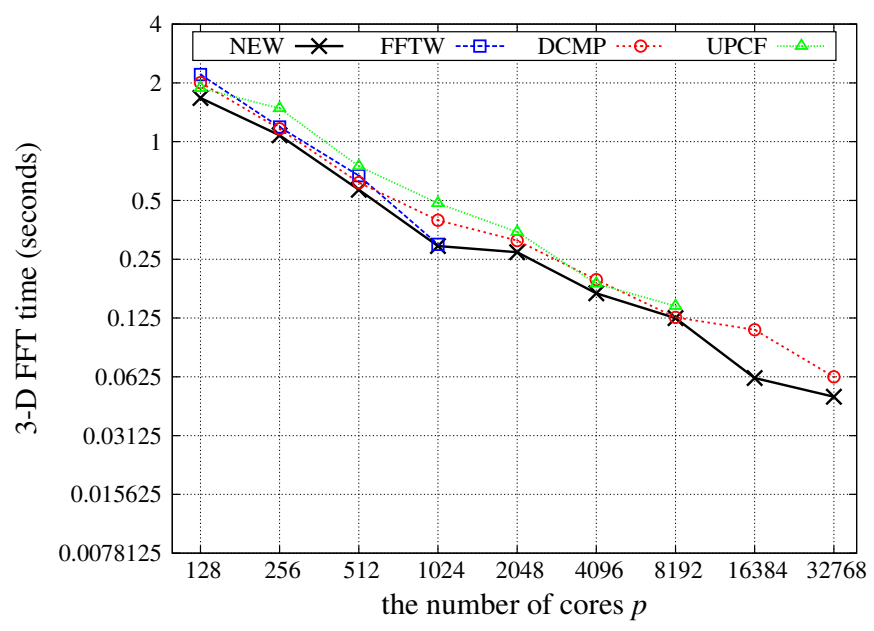

(b) Hopper (Strong Scaling)

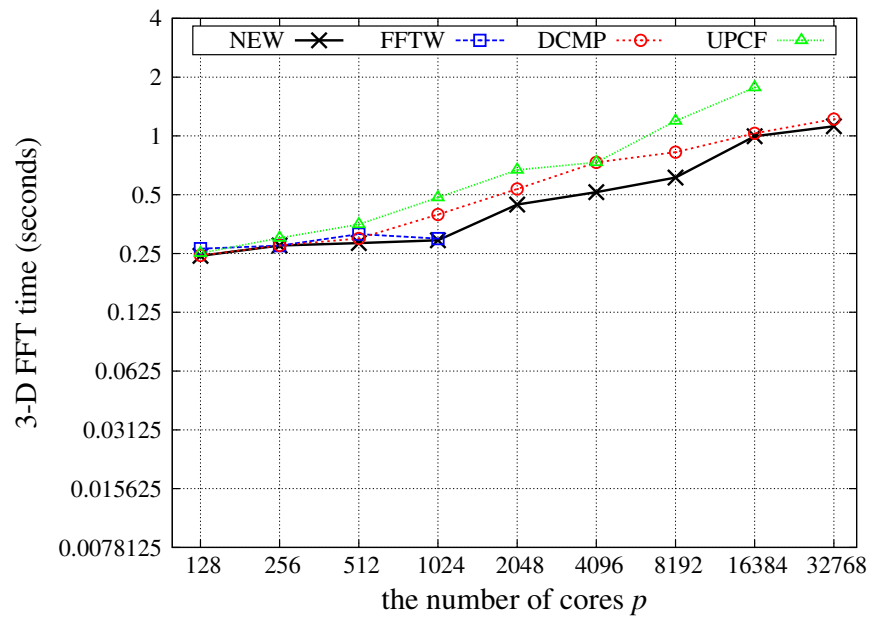

(d) Hopper (Weak Scaling)

Figure 7: Parallel 3-D FFT Execution Time on Large Scale Experiments (log-log Scale Plot): Strong scaling fixes an input size at 1024 ${ }^{3}$ elements. Weak scaling doubles an input size from $512^{3}$ through $4096 \times 4096 \times 2048$. NEW scales up to at least 32,768 cores while FFTW only scales up to 1,024 cores.

tion for Edison is not the best on Hopper, and vice versa. Thus, it is necessary to auto-tune our 3-D FFT code for each different platform to achieve the best of its performance.

\subsection{Large Scale Experiments}

For strong scaling experiments, we increase $p$ from 128 through 32,768 cores (around a quarter of the total cores), and we have a fixed-size input array with $1024^{3}$ elements. The third column of Table 3(a) shows the 3-D FFT execution time of our NEW approach on Edison. Figure 6(a) shows the speedup of our NEW approach relative to three other approaches on Edison. NEW shows speedup of up to $1.29 \times$ over FFTW, $2.57 \times$ over DCMP, and $1.36 \times$ over UPCF. Note that FFTW only scales up to 1,024 cores as FFTW is based on 1-D decomposition. On the other hand, NEW, DCMP, and UPCF scale to at least 32,768 cores as they are all based on 2-D decomposition. Figures 7(a) and 8(a) show the parallel 3-D FFT execution time and the FLOPS performance for strong scaling experiments on Edison. The execution time of NEW decreases from 0.965 seconds to 0.012 seconds. We also computed FLOPS performance values based on the rule of $5 \mathrm{~N} \log _{2} \mathrm{~N}$ floating point operations for 1-D FFT on $N$ elements. 9] The FLOPS performance of NEW ranges from 167 GFLOPS to 13,603 GFLOPS, which means the parallel efficiency is $31.8 \%$ going from 128 to 32,768 cores. The strong scaling performance increases sub-linearly as parallel 3-D FFT involves heavy overhead of global communication patterns at large $p$.

In weak scaling experiments, $p$ increases from 128 to 32,768 cores, and the input size also doubles from $512^{3}$ through $4096 \times 4096 \times 2048$. The fifth column of Table 3(c) shows the 3 -D FFT execution time of NEW on Edison. Figures 6(c) 7(c) and 8(c) show the results of weak scaling experiments on Edison. The speedup of NEW 


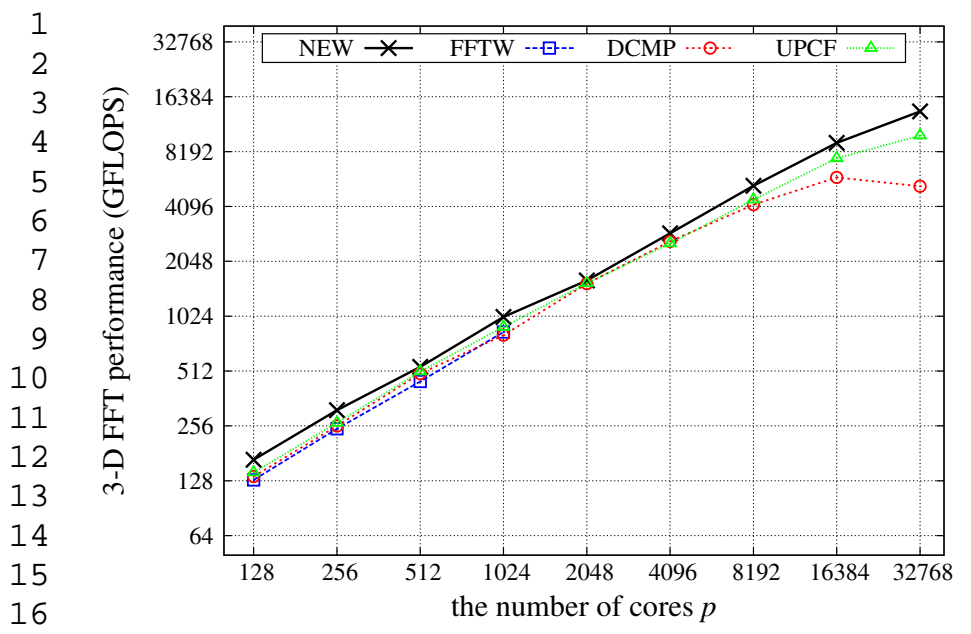
5,365 GFLOPS (weak scaling) at 32,768 cores. is up to $1.35 \times$ over FFTW, $1.26 \times$ over DCMP, and $1.34 \times$ over UPCF. The FFT execution time of NEW increases from 0.114 seconds to 0.341 second. The curves in Figure 7(c) are increasing with $p$ while they are supposed to be flat in the ideal weak scaling. One reason of the nonflat curve is the overhead of all-to-all communication. The other reason is because the computation complexity of 3-D FFT grows faster than an input size. For an input with $N_{x} \times N_{y} \times N_{z}$ elements, we need $O\left(N_{x} N_{y} N_{z} \log \left(N_{x} N_{y} N_{z}\right)\right)$ floating point operations. The FLOPS performance of NEW ranges between 159 GFLOPS and 17,611 GFLOPS, which means the parallel efficiency is $43.3 \%$ going from 128 to 32,768 cores. The parallel efficiency of weak scaling is higher than strong scaling because weak scaling has less communication overhead with larger-size messages than strong scaling.

Likewise, we did the same experiments for strong scal- (a) Edison (Strong Scaling)

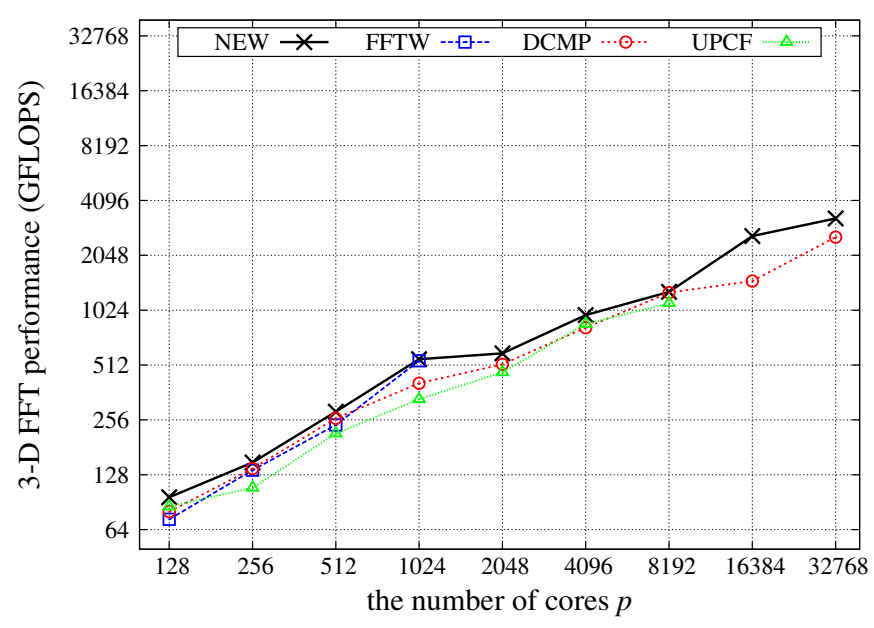

(b) Hopper (Strong Scaling)

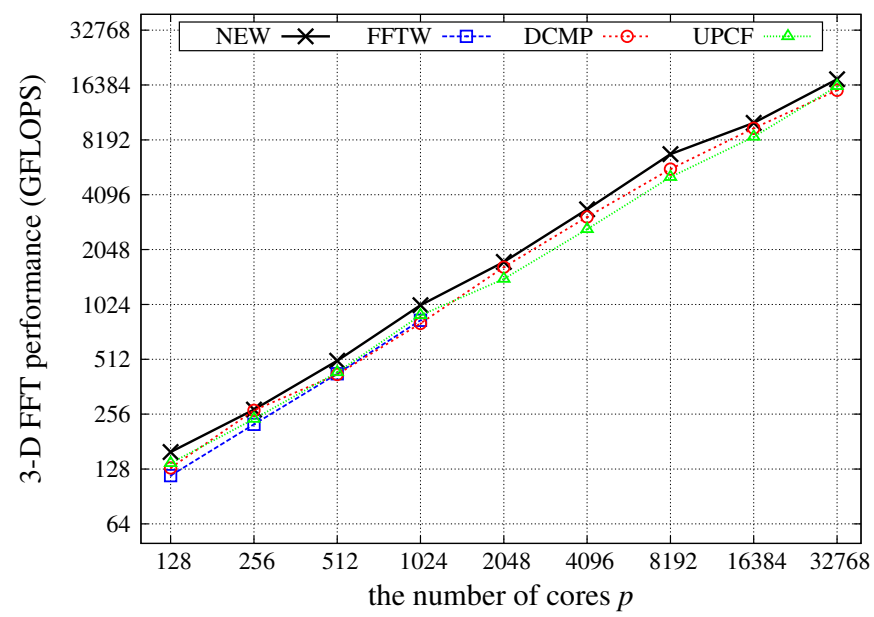

(c) Edison (Weak Scaling)

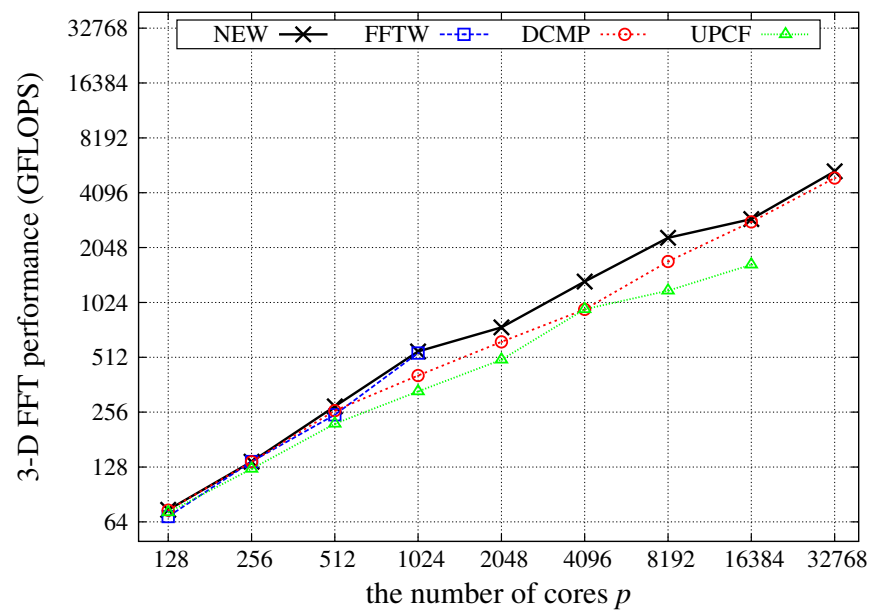

(d) Hopper (Weak Scaling)

Figure 8: Parallel 3-D FFT Performance in GFLOPS on Large Scale Experiments (log-log Scale Plot): Strong scaling fixes an input size at $1024^{3}$ elements. Weak scaling doubles an input size from $512^{3}$ through $4096 \times 4096 \times 2048$. NEW achieves 13,603 GFLOPS (strong scaling) and 17,611 GFLOPS (weak scaling) at 32,768 cores on Edison. The performance of NEW on Hopper is 3,262 GFLOPS (strong scaling) and

ing and weak scaling on Hopper. Table 3(b) and Figures 6(b) 7(b) and 8(b) show the results of strong scaling experiments on Hopper. NEW shows speedup of up to $1.32 \times$ over FFTW, $1.77 \times$ over DCMP, and $1.66 \times$ over UPCF. (UPCF crashed at 16,384 and 32,768 cores after producing an unknown error.) The execution time of NEW decreases from 1.673 seconds to 0.049 seconds. The FLOPS performance increases from 96 GFLOPS to 3,262 GFLOPS, which means the parallel efficiency is $13.2 \%$ going from 128 to 32,768 cores. Table 3(d) and Figures 6(d) $7(\mathrm{~d})$ and $8(\mathrm{~d})$ show the results of weak scaling experiments on Hopper. NEW shows speedup of up to $1.11 \times$ over FFTW, $1.42 \times$ over DCMP, and $1.95 \times$ over UPCF. (UPCF crashed at 32,768 cores after producing an unknown error.) The execution time of NEW increases from 0.243 seconds to 1.121 second. The FLOPS performance increases from 75 GFLOPS to 5,365 GFLOPS, which means the parallel 


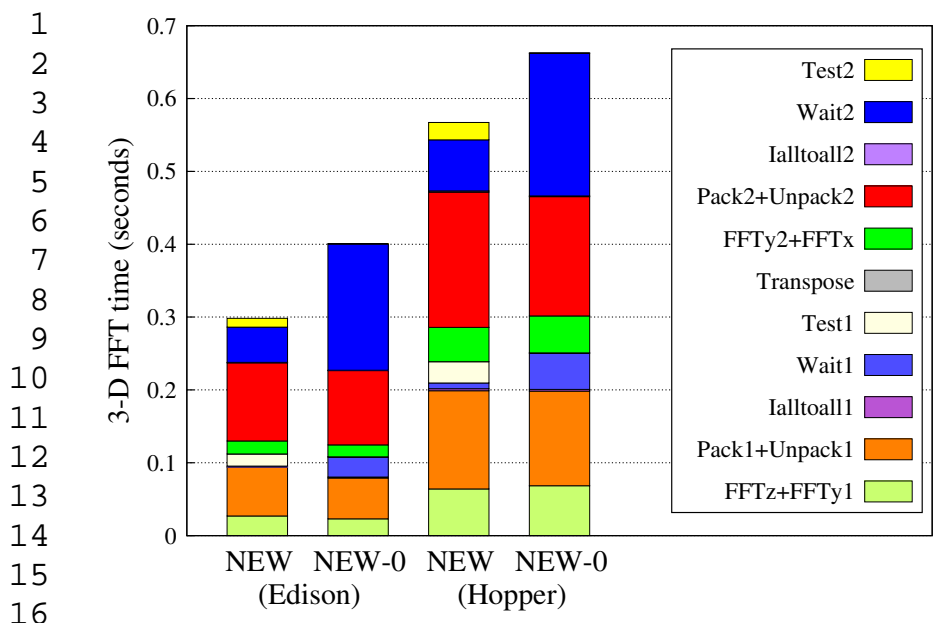

(a) Small Scale $\left(p=512, N^{3}=1024^{3}\right)$

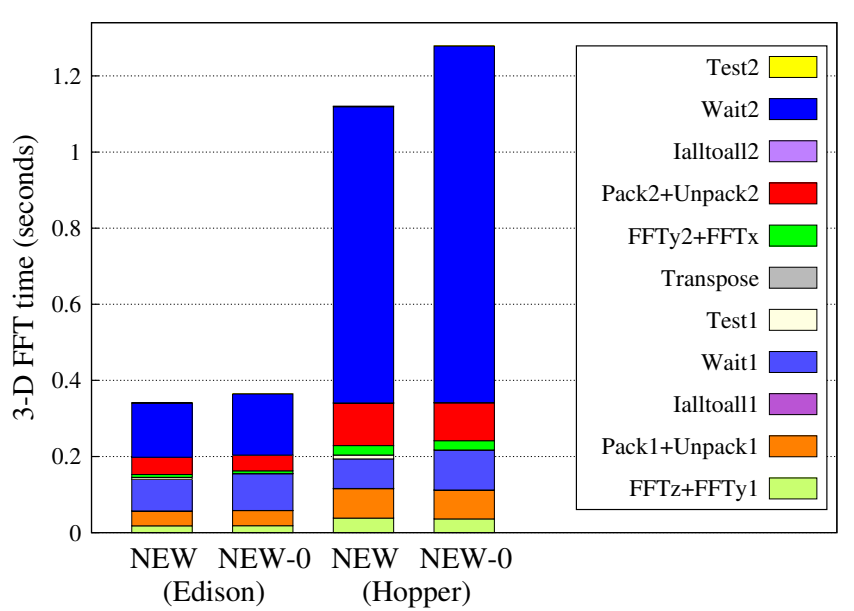

(b) Large Scale $\left(p=32768, N_{x} \times N_{y} \times N_{z}=4096 \times 4096 \times 2048\right)$

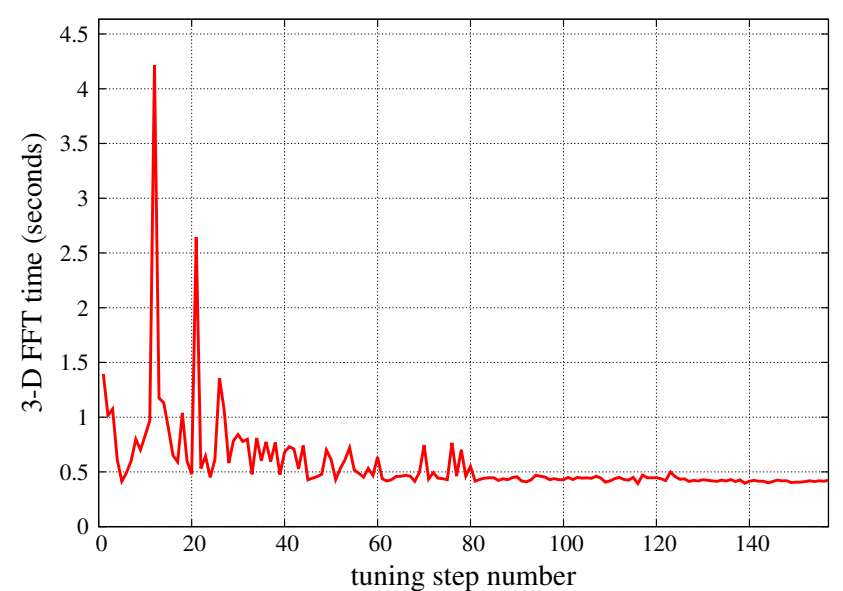

(a) Small Scale $\left(p=512, N^{3}=1024^{3}\right)$

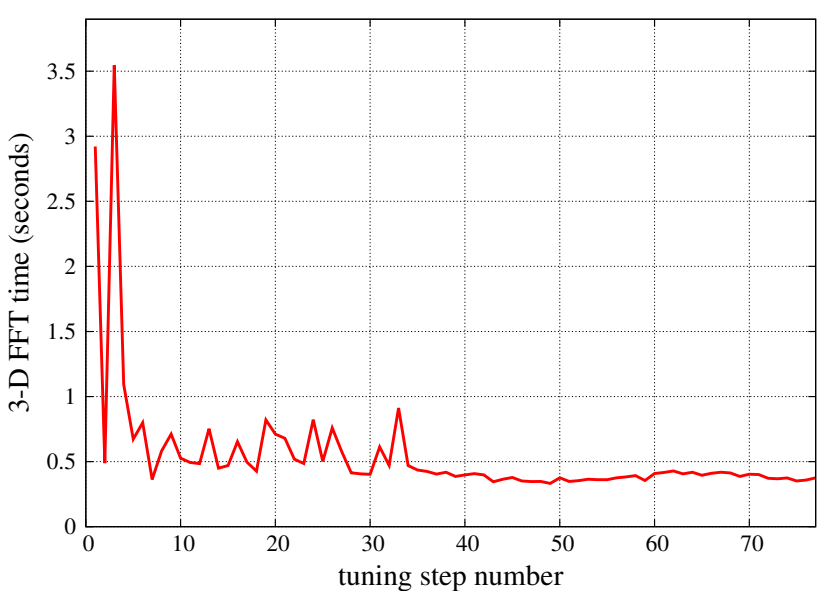

(b) Large Scale $\left(p=32768, N_{x} \times N_{y} \times N_{z}=4096 \times 4096 \times 2048\right)$

Figure 10: Auto-Tuning History on Edison: The 3-D FFT time for a test parameter configuration decreases as the tuning proceeds. There is around $10 \times$ performance gap between the best and the worst configurations. Thus we should tune the parameters properly to achieve high performance of 3-D FFT.

efficiency is $28.1 \%$ going from 128 to 32,768 cores.

In large-scale experiments, NEW is still faster than FFTW, DCMP, and UPCF for almost all different input settings. As we discussed in the previous section, the successful computation-communication overlap is the reason of NEW's high performance. For example, Figure 9(b) shows the breakdown of the execution time of NEW for the $p=32768$ and $N_{x} \times N_{y} \times N_{z}=4096 \times 4096 \times 2048$ configuration. On Edison and Hopper, NEW reduces communication time (Wait1 and Wait2) compared to the nonoverlapped version NEW-0. Table B] contains the autotuned parameter configurations of NEW, which shows that the best parameter values vary depending on system settings. Figure 10(b) shows the history of an auto-tuning execution for one setting on Edison. There is around $10 \times$ performance gap between the best and the worst configu- rations. We could achieve high performance of 3-D FFT in large-scale experiments by utilizing the auto-tuning technique.

The FFT performance on Edison is faster than Hopper for all input settings. Edison is a newer machine with faster inter-connection network and higher computation performance than Hopper. Also, the parallel efficiency on Edison is higher than Hopper. The Dragonfly network of Edison [18, 19] connects compute nodes with short hop distances which is beneficial for all-to-all communication, and the dynamic routing mechanism balances network traffic over inter-connection links. Thus Edison handles largescale global network traffic better than the 3-D Torus network of Hopper. 


\subsection{Tuning Costs}

In order to quantify the cost of auto-tuning, we collected all the history of 270 auto-tuning executions of NEW that generated the results in Tables [2 and 3] Figure [1] shows the distribution of the number of tuning steps taken by each tuning execution. $y$-axis means the cumulative fraction of tuning executions, and $x$-axis represents the number of tuning steps. The curve marked with "converge" represents the distribution of the total number of tuning steps that each tuning execution takes until the Nelder-Mead algorithm converges. The curve marked with "best" represents the distribution of the number of tuning steps that each tuning execution takes until finding the best configuration. Figure [1] shows that our method uses 121 steps until convergence in the median case $(y=0.5)$, and the best configuration is found after 92 steps. Note that scientific simulations normally execute more than tens of thousands of time steps, each of which involves a couple of 3-D FFT operations. Thus our auto-tuning cost of around 100 3-D FFT operations will be almost negligible in such scientific simulations. This low cost of tuning is the result of our customization techniques described in Section 4.3

Despite the already low tuning cost, we can reduce it even more by sacrificing a little bit of 3-D FFT performance. The other curves in Figure 11 represent the number of tuning steps that each tuning execution takes until finding a configuration close to the best one. For example, the curve of " $5 \%$ worse" is for a configuration such that the 3 -D FFT time takes $5 \%$ more than the best configuration. It is interesting that we can still find a good configuration by shortening many tuning steps. For example, we can find a " $5 \%$ worse" configuration after 41 steps (median), which is faster than 92 steps for the "best" configuration by more than a factor of two. Thus we can bring down the 3-D FFT performance slightly and shorten the number of tuning steps significantly.

\section{Related Work}

FFTW 9] does not overlap any computation with communication, so it results in the poor performance of 3-D FFT as shown in Section 5. Also, FFTW is not scalable as it is designed based on 1-D decomposition. P3DFFT [4], 2DECOMP\&FFT [5], Ayala et al. 6], Takahashi 7], and Eleftheriou et al. 20] achieved high scalability with the high-dimensional domain decomposition technique. However, they did not include any overlap between computation and communication. On the other hand, our approach increases both of the performance and scaling through computation-communication overlap and 2-D decomposition.

Doi et al. 21] overlap computation and communication in a shared-memory parallel environment. However, each core uses blocking communication, and the overlap takes place between cores. On the other hand, we exploit non-blocking communication for the overlap within

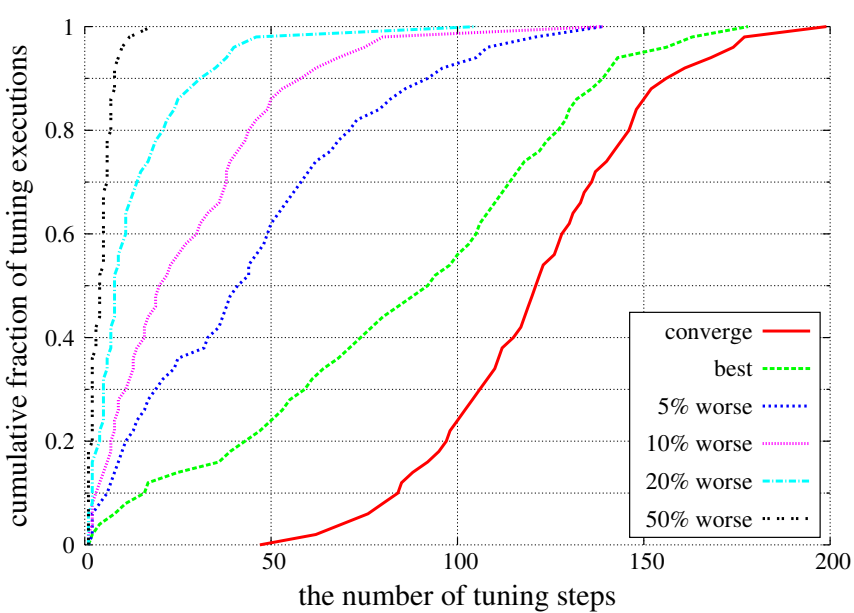

Figure 11: Cumulative Distribution of the Number of Tuning Steps: In the median case $(y=0.5)$, the "best" configuration is found after 92 steps, which is almost negligible in scientific simulations executing more than tens of thousands of 3-D FFT operations. We can reduce the tuning cost significantly by sacrificing a little bit of 3-D FFT time. For example, a " $5 \%$ worse" configuration is found after 41 steps.

each core. Bell et al. [13] and Nishtala et al. 8] wrote a UPC-based code that requires hardware support for asynchronous communication. As shown in Section 5 the UPCbased code is not optimized because 1) it misses some computation to overlap with communication, 2) it uses a fixed size of communication messages, and 3) it uses the point-to-point communication rather than the optimized collective operation. Fang et al. 22 lack portability as they use a special communication API called QMP and require hardware support for asynchronous communication. Also, there is no auto-tuning of overlap-related parameters. Similarly to our approach, Hoefler et al.'s work [10] and our previous work 11] succeeded in using MPI_Ialltoall for 3-D FFT, but they have limitations on scalability as they are based on 1-D decomposition. Note that our algorithm also supports 1-D decomposition through auto-tuning.

Kandalla et al. 23] overlap the computation on one input array with the communication for other arrays. This "inter-array" overlap is useful when there are many independent arrays for 3-D FFT. However, scientific simulations 1] normally need successive 3-D FFT computations over time on a single array. In this case, our "intra-array" method is effective as we overlap computation with communication inside each 3-D FFT operation. Also, Kandalla et al.'s approach requires hardware support for asynchronous communication.

\section{Conclusions and Future Work}

This paper presented a scalable method for parallel 3D FFT that exploits computation-communication overlap. Our work is the first to effectively apply non-blocking MPI collectives to the 2-D decomposition method for parallel 
3-D FFT. We need no special hardware support for asynchronous communication. We also described a method to auto-tune our 3-D FFT code in a large parameter space. Experimental results show that our code scales well up to at least 32,768 cores, and performs faster than three other approaches including the UPC-based code. Our auto-tuning technique is effective as two systems require different settings of the best parameter configuration.

We are planning to extend this work by improving the auto-tuning method. The quality and speed of the Nelder-Mead heuristic is dependent on how the initial simplex is defined. Although our definition of the initial simplex was successful, it is worth investigating if there exist other more effective initial simplex construction techniques. Also, we plan to try optimization strategies other than Nelder-Mead.

\section{Acknowledgments}

Support for this work was provided through Scientific Discovery through Advanced Computing (SciDAC) program funded by U.S. Department of Energy, Office of Science, Advanced Scientific Computing Research under award numbers ER25763 and ER26054. This research used resources of the National Energy Research Scientific Computing Center, a DOE Office of Science User Facility supported by the Office of Science of the U.S. Department of Energy under Contract No. DE-AC02-05CH11231.

\section{References}

[1] T. Ishiyama, K. Nitadori, J. Makino, 4.45 pflops astrophysical n-body simulation on k computer: the gravitational trillionbody problem, in: Proceedings of the 2012 ACM/IEEE International Conference for High Performance Computing, Networking, Storage and Analysis (SC), IEEE Computer Society Press, 2012.

[2] M. Lee, N. Malaya, R. D. Moser, Petascale direct numerical simulation of turbulent channel flow on up to $786 \mathrm{k}$ cores, in: Proceedings of the $2013 \mathrm{ACM}$ /IEEE International Conference for High Performance Computing, Networking, Storage and Analysis (SC), ACM Press, 2013.

[3] S. Song, J. K. Hollingsworth, Scaling parallel 3-d FFT with non-blocking MPI collectives, in: Proceedings of the 5th Workshop on Latest Advances in Scalable Algorithms for Large-Scale Systems (ScalA), 2014, pp. 1-8.

[4] D. Pekurovsky, P3DFFT: A Framework for Parallel Computations of Fourier Transforms in Three Dimensions, SIAM Journal on Scientific Computing 34 (4).

[5] N. Li, S. Laizet, 2decomp \& fft-a highly scalable 2d decomposition library and fft interface, in: Cray User Group 2010 conference, 2010.

[6] O. Ayala, L.-P. Wang, Parallel implementation and scalability analysis of $3 \mathrm{~d}$ fast fourier transform using $2 \mathrm{~d}$ domain decomposition, Parallel Computing 39 (1).

[7] D. Takahashi, An implementation of parallel 3-d fft with 2d decomposition on a massively parallel cluster of multi-core processors, in: Parallel Processing and Applied Mathematics, Vol. 6067 of Lecture Notes in Computer Science, Springer Berlin Heidelberg, 2010.

[8] R. Nishtala, P. Hargrove, D. Bonachea, K. A. Yelick, Scaling communication-intensive applications on bluegene/p using one-sided communication and overlap, in: Proceedings of the 23rd International Parallel \& Distributed Processing Symposium (IPDPS), 2009, pp. 1-12.

[9] M. Frigo, S. G. Johnson, The design and implementation of FFTW3, Proceedings of the IEEE 93 (2), special issue on "Program Generation, Optimization, and Platform Adaptation".

[10] T. Hoefler, P. Gottschling, A. Lumsdaine, Brief announcement: Leveraging non-blocking collective communication in high-performance applications, in: Proceedings of the 20th annual symposium on Parallelism in algorithms and architectures (SPAA), ACM, 2008.

[11] S. Song, J. K. Hollingsworth, Designing and auto-tuning parallel 3-d fft with computation-communication overlap, in: Proceedings of the ACM SIGPLAN Symposium on Principles and Practice of Parallel Programming (PPoPP), ACM Press, 2014.

[12] M. P. I. Forum, Mpi: A messagepassing interface standard version 3.0, http://www.mpi-forum.org/docs/mpi-3.0/mpi30-report.pdf

[13] C. Bell, D. Bonachea, R. Nishtala, K. Yelick, Optimizing bandwidth limited problems using one-sided communication and overlap, in: Proceedings of the 20th International Parallel \& Distributed Processing Symposium (IPDPS), IEEE Computer Society Press, 2006.

[14] C. Tुăpuş, I.-H. Chung, J. K. Hollingsworth, Active harmony: towards automated performance tuning, in: Proceedings of the $2002 \mathrm{ACM} / \mathrm{IEEE}$ International Conference for High Performance Computing, Networking, Storage and Analysis (SC), ACM Press, 2002.

[15] T. Hoefler, A. Lumsdaine, Message progression in parallel computing - to thread or not to thread?, in: Proceedings of the 2008 IEEE International Conference on Cluster Computing (CLUSTER), 2008

[16] J. A. Nelder, R. Mead, A simplex method for function minimization, The Computer Journal 7 (4).

[17] E. Brachos, Parallel fft libraries, Master's thesis, The University of Edinburgh (2011).

[18] J. Kim, W. J. Dally, S. Scott, D. Abts, Technology-driven, highly-scalable dragonfly topology, in: Proceedings of the 35th International Symposium on Computer Architecture (ISCA), 2008.

[19] G. Faanes, A. Bataineh, D. Roweth, T. Court, E. Froese, R. Alverson, T. Johnson, J. Kopnick, M. Higgins, J. Reinhard, Cray cascade: a scalable HPC system based on a dragonfly network, in: Proceedings of the 2012 ACM/IEEE International Conference for High Performance Computing, Networking, Storage and Analysis (SC), IEEE Computer Society Press, 2012.

[20] M. Eleftheriou, B. G. Fitch, A. Rayshubskiy, T. J. C. Ward, R. S. Germain, Scalable framework for $3 \mathrm{~d}$ ffts on the blue gene/1 supercomputer: implementation and early performance measurements, IBM J. Res. Dev. 49 (2).

[21] J. Doi, Y. Negishi, Overlapping methods of all-to-all communication and fft algorithms for torus-connected massively parallel supercomputers, in: Proceedings of the 2010 ACM/IEEE International Conference for High Performance Computing, Networking, Storage and Analysis (SC), IEEE Computer Society Press, 2010.

[22] B. Fang, Y. Deng, G. J. Martyna, Performance of the 3d fft on the $6 \mathrm{~d}$ network torus qcdoc parallel supercomputer, Computer Physics Communications 176 (8).

[23] K. Kandalla, H. Subramoni, K. Tomko, D. Pekurovsky, S. Sur, D. K. Panda, High-performance and scalable non-blocking allto-all with collective offload on infiniband clusters: a study with parallel 3d fft, Computer Science 26 (3-4). 